\title{
Photodissociation Dynamics
}

\author{
Laurie J. Butler* \\ Department of Chemistry, University of Chicago, Chicago, Illinois 60637
}

\begin{abstract}
Daniel M. Neumark*
Department of Chemistry, University of California, Berkeley, California 94720, and Chemical Sciences Division, Lawrence Berkeley National Laboratory, Berkeley, California 94720
\end{abstract}

Received: November 28, 1995; In Final Form: March 25, $1996^{\otimes}$

\begin{abstract}
Advances in the study of photodissociation dynamics over the past 30 years are reviewed. An overview of experimental techniques that have been developed to extract photofragment energy and angular distributions is presented, followed by a discussion on several current topics of interest in the field of photodissociation.
\end{abstract}

\section{Introduction}

The field of photodissociation dynamics has grown explosively in the past few decades. Spearheaded by developments in laser and molecular beam techniques, studies of photodissociation dynamics now pervade virtually all areas of physical chemistry from environmental chemistry, through cluster and gas-surface phenomena, to quantum scattering theory. Photodissociation experiments provide some of the most critical tests of the fundamental assumptions we make in calculating the rates of chemical reactions via transition state theories: the assumption of the separability of nuclear and electronic motion and the assumption of rapid intramolecular vibrational energy redistribution (IVR). They also speak to immediate societal issues such as the destruction of the ozone layer due to chlorofluorocarbon pollutants.

This review seeks to outline the most important experimental advances in the field of photodissociation dynamics and to explore how the experiments deepen the way we think about chemical reaction dynamics. In this analysis, we show how studies of photodissociation dynamics speak to the core of our understanding of a wide variety of phenomena, allowing us to probe the very nature of an elementary chemical reaction: the breaking and forming of chemical bonds. The first section focuses on the incredible variety of experimental techniques that have been developed over the years to characterize the product state distributions arising from photodissociation. The second section discusses several more specific applications of photodissociation, with particular emphasis on dynamical processes that primarily occur on excited state potential energy surfaces. In both sections, we omit reviewing real-time experiments, ground state photodissociation dynamics, and photodissociation of clusters and ions as these topics are covered in reviews elsewhere in this issue.

Due to space limitations, the authors have focused primarily on experimental rather than theoretical studies and have nevertheless not included numerous important experiments. We hope our valued colleagues will forgive us for concentrating on the limited selection of papers reviewed below. We also point out that more detailed descriptions of several experimental methods mentioned in this article can be found in the review article by Houston in this volume.

${ }^{\otimes}$ Abstract published in Advance ACS Abstracts, June 15, 1996.

\section{The Development of Experimental Tools in Photodissociation Dynamics}

A. Overview and Motivation. One of the driving forces in the field of chemical dynamics has been to perform increasingly state selective experiments, in which as many quantum numbers as possible of both the reactant and product are specified. Experiments of this type are most readily compared to detailed theoretical calculations and therefore result in significant advances in our understanding of elementary chemical processes. Photodissociation experiments are exceptionally appealing from this perspective, because in contrast to bimolecular scattering experiments, one can often specify the reactant energy and angular momentum. In order to take full advantage of this capability, one would like to completely characterize the products resulting from photodissociation. In this way one learns how the initial photoexcitation of the reactant molecule is channeled into the multitude of product degrees of freedom. Such a characterization is the goal underlying the various experimental techniques described in this section. As should be clear from the following discussion, no single technique is ideal for all photodissociation experiments. Each represents a compromise between generality, sensitivity, and resolution. Collectively, they provide a powerful experimental methodology with which to attack and understand photodissociation dynamics.

B. Photofragment Velocity and Angular Distribution Measurements. Molecular beam photofragment translational energy spectroscopy offered the first opportunity to study the dynamics of photodissociation reactions in a collision-free regime. The authors of the first experimental work of this kind ${ }^{1}$ comment in one of their early articles, ${ }^{2}$ "We believe that ... the molecular dynamics of photodissociation offer a fertile and largely untilled testing ground for theories of unimolecular processes, and that much of the knowledge gained will also be applicable to bimolecular processes such as energy transfer and chemical reaction". This prophetic statement is borne out by the studies reviewed in section III of this article.

From the first instruments for measuring photofragment translational energy ${ }^{3}$ and angular distributions ${ }^{4,5}$ to the recent high-resolution $\mathrm{H}$ atom Rydberg time-of-flight spectrometer, ${ }^{6}$ the experiments seek to measure two observables: (1) the time required for a photofragment, produced in the center of a molecular beam apparatus by a pulsed, usually narrow-band, photolysis laser, to travel from the interaction region of the laser 
and molecular beam to the detector and (2) the angular distribution ${ }^{7}$ of the photofragments with respect to the electric vector of the dissociating laser. As in the first apparatus of Wilson's, the detector in many current instruments designed to measure photofragment velocity and angular distributions consists of an electron-bombardment ionizer followed by a mass spectrometer. This configuration can detect any neutral photofragment, whether stable molecule or reactive radical, irrespective of its internal energy or other quantum state, and is thus termed a universal detector. ${ }^{8}$

The measured translational energy of the photofragment provides critical dynamical information. The recoil velocities of the two fragments of a $\mathrm{A} \rightarrow \mathrm{B}+\mathrm{C}$ dissociation event must be related by momentum conservation, so at the very least a measurement of the velocity of one photofragment, when converted to the center-of-mass reference frame, completely determines the velocity distribution of the other photofragment and the distribution of energy partitioned to relative recoil kinetic energy in the dissociation. If the dissociation produces an atom (in just one electronic state) plus a radical, conservation of energy requires that the internal energy of the radical is also completely determined (if the parent internal energy is adequately cooled in the supersonic expansion). Thus, the I atom time-of-arrival spectrum in the photodissociation of $\mathrm{CH}_{3} \mathrm{I}$ reveals a progression of peaks that reflect the vibrational quantum state of the momentum-matched $\mathrm{CH}_{3}$ partner radical, showing the $\mathrm{CH}_{3}$ partner is produced with a distribution of quanta in umbrella bending motion.

One of the more interesting examples of determining the quantum state distribution of the radical partner fragment by detecting the atomic fragment's velocity is in recent $\mathrm{H}$ atom Rydberg time-of-flight measurements on $\mathrm{H}_{2} \mathrm{~S}$ photodissociation. This technique foregoes the universal detector but offers spectacular resolution. ${ }^{6}$ In the $121.6 \mathrm{~nm}$ photodissociation of $\mathrm{H}_{2} \mathrm{~S}$ to $\mathrm{H}+\mathrm{SH}(\tilde{\mathrm{A}})$ shown in Figure 1 , the $\mathrm{H}$ atom time-offlight spectrum reveals peaks corresponding to each rovibrational state of the momentum-matched $\mathrm{SH}(\tilde{\mathrm{A}})$ diatomic. The $\mathrm{SH}$ rovibronic levels reflected in the $\mathrm{H}$ atom flight times are highly predissociative, so they could not have been detected with laser probes of the $\mathrm{SH}$ radical! Clearly the primary $\mathrm{S}-\mathrm{H}$ bond fission is essentially complete before the $\mathrm{SH}$ product predissociates.

While photofragment velocity distributions do not often carry detailed quantum state specific information as in the nice examples described above, they do give key information on some features of the reactive potential energy surface. For instance, in reactions where there is a large barrier to the reverse reaction, the recoil on this repulsive region of the potential after the barrier often results in considerable energy being partitioned into product translation, resulting in a product translational energy distribution that is peaked well away from zero kinetic energy. Thus, even if the excited state and ground state potential energy surfaces correlate to the same dissociation asymptote, if there is no exit barrier along the ground state reaction coordinate but a significant one along the excited state reaction coordinate, the photofragment velocity distribution will determine whether the mechanism for the reaction involved internal conversion or not. Likewise, the photofragment angular distributions ${ }^{7}$ can provide a wealth of information. Angular distributions from a single photon absorption event where the rotational quantum state of the parent and photofragment are not resolved are traditionally used to provide a rough measure of the time scale of the dissociation event. An isotropic distribution signals that the dissociation time was long with respect to a rotational period. The distribution, if anisotropic, reflects the direction of the electronic dipole moment operative
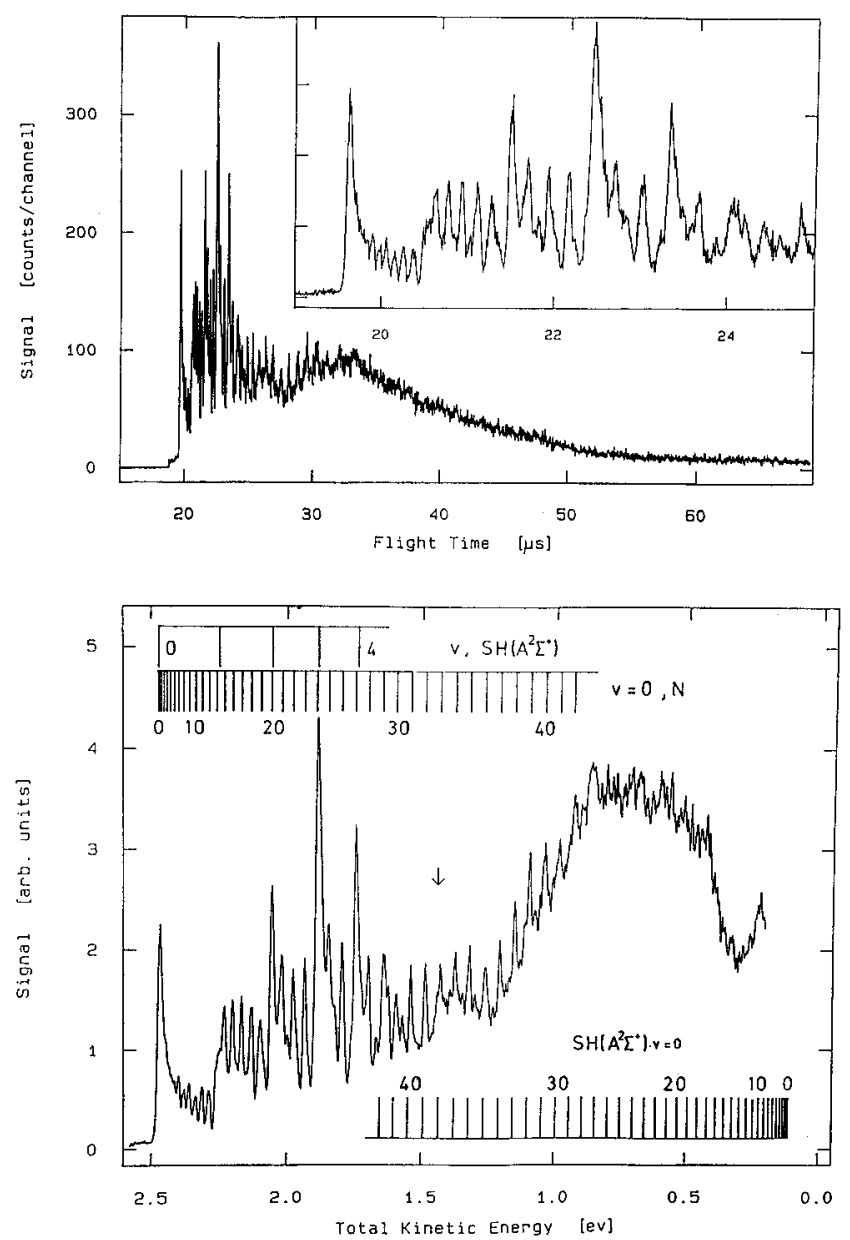

Figure 1. Product quantum state resolution with the $\mathrm{H}$ atom Rydberg TOF technique. Top frame: $\mathrm{H}$ atom flight times from the photodissociation of $\mathrm{H}_{2} \mathrm{~S}$ at $121.6 \mathrm{~nm}$. Bottom frame: Total kinetic energy spectrum, derived from the data in the top frame for $\mathrm{H}$ atoms recoiling from $\mathrm{SH}$ fragments. The structure in the kinetic energy distribution arising from the rovibrational quantum state of the $\mathrm{SH}(\mathrm{A})$ fragment is assigned in the comb above the spectrum. The possible kinetic energies of the $\mathrm{H}$ and $\mathrm{S}\left({ }^{3} \mathrm{P}\right)$ fragments resulting from the subsequent predissociation of these SH(A) fragments are shown in the comb below the spectrum. Adapted with permission from Figures 1 and 2 in ref 6 .

in the absorption that resulted in a given photofragmentation channel. ${ }^{9}$ In more recent experiments angular distributions have been used to identify whether a given photofragmentation channel occurs preferentially from one of two thermally populated molecular conformers. ${ }^{10}$

Experiments using the technique of photofragment translational energy spectroscopy have played a key role in testing and developing our understanding of statistical theories of chemical reaction dynamics and in probing the nature of forces at the transition state and in the exit channel of chemical reactions; sections III.C, III.D.1, and III.D.2 detail several specific examples and refer to some useful review articles. Such experiments have also offered the opportunity to probe the influence of electronically nonadiabatic effects on the branching between energetically allowed dissociation channels; examples are included in section III.D.3. Overall, photofragment velocity and angular distribution analysis is a crucial tool in studying the dynamics of photodissociation reactions, providing complementary data to optical probes of the quantum state distributions of the photofragments described in the next section.

C. State-Resolved Detection of Photofragments. The photofragment translational spectroscopy methods described above have the advantage of great generality. However, with 
the exception of the $\mathrm{H}$ atom time-of-flight experiments, they typically do not have sufficient energy resolution to yield information on the rotational distribution of the photofragments. This is a significant issue, since rotational distributions often yield an intuitive picture of the microscopic forces involved in photodissociation. For example, one can infer whether the energy available to the products is released in linear or bent molecular configurations from the extent of rotational excitation in the fragments.

These considerations have spurred the development of statespecific spectroscopic probes of photofragments during the past 20 years. In this section we will attempt to summarize the vast amount of work in this area, with emphasis on those techniques that yield rotational resolution. We first consider measurements whose primary purpose is to determine the nascent rotational and vibrational energy distribution in a single fragment. This will be followed by a discussion of correlated measurements, particularly those in which the velocity and/or angular distribution of state-selected fragments is measured.

1. Laser-Induced Fluorescence. The measurement of photofragment energy distributions with laser-induced fluorescence (LIF) has become the single most common type of photodissociation experiment. Such an experiment requires that the fragment of interest has an electronic transition accessible with a pulsed dye laser (through either a one- or two-photon transition) and that the spectroscopy of this transition is wellcharacterized. The first experiments of this type were performed in the late 1970s. In a series of experiments carried out in several laboratories, laser-induced fluorescence was used to probe the $\mathrm{CN}$ photofragment from $\mathrm{ICN}$ and $\mathrm{C}_{2} \mathrm{~N}_{2}$ photolysis, ${ }^{11,12}$ the $\mathrm{CH}_{2}\left(\mathrm{a}^{1} \mathrm{~A}_{1}\right)$ fragment from photodissociation of $\mathrm{CH}_{2} \mathrm{CO},{ }^{13,14}$ and the $\mathrm{NH}\left(\mathrm{a}^{1} \Delta\right)$ fragment from $\mathrm{HN}_{3}$ and $\mathrm{HNCO} .{ }^{15,16}$ Experiments performed shortly thereafter probed CS production from $\mathrm{CS}_{2},{ }^{17} \mathrm{SH}$ production from $\mathrm{H}_{2} \mathrm{~S},{ }^{18}$ the $\mathrm{NO}$ fragment from $\mathrm{CF}_{3}-$ $\mathrm{NO}$ and $\mathrm{NO}_{2}$ dissociation, ${ }^{19,20}$ the $\mathrm{CN}$ and $\mathrm{NO}$ fragments from NCNO photodissociation, ${ }^{21}$ and the $\mathrm{OH}$ fragment from dissociation of $\mathrm{H}_{2} \mathrm{O},{ }^{22} \mathrm{H}_{2} \mathrm{O}_{2},{ }^{23}$ and $\mathrm{HONO}^{24}$ In several of these studies, photofragment spin-orbit and $\Lambda$-doublet populations were extracted in addition to the rotational and vibrational distributions, thereby providing further insight into the dissociation dynamics.

Most of these studies were carried out in room temperature gas cells, so that the internal energy distribution of the photofragments includes the initial thermal energy spread of the reactant molecules. In the early 1980s, the first photodissociation/LIF experiments were performed in which the reactants were cooled in a pulsed free jet expansion. The results for $\mathrm{H}_{2} \mathrm{~S}^{25}$ and $\mathrm{H}_{2} \mathrm{O}^{22}$ photodissociation showed cooling of the $\mathrm{SH}$ and $\mathrm{OH}$ rotational distribution compared to gas cell experiments; for jetcooled $\mathrm{H}_{2} \mathrm{O}$, a significant inversion in the $\mathrm{OH} \Lambda$-doublet population was observed as well. Similar trends were seen in the $\mathrm{CN}$ and $\mathrm{NO}$ rotational distributions produced from photolysis of jet-cooled NCNO. ${ }^{26}$ These experiments clearly demonstrate the advantage of cooling the reactant molecules when trying to extract dynamics from product state distributions, particularly when the photolysis band is unstructured.

The development of vacuum-ultraviolet lasers that operate in the 1300-1500 $\AA$ range enabled the investigation of CO fragments from photodissociation via the $\mathrm{A}^{1} \Pi \leftarrow \mathrm{X}^{1} \Sigma^{+}$transition, thereby considerably extending the range of molecules in which photodissociation could be studied by LIF. In the first experiments of this type, rotationally resolved $\mathrm{CO}$ distributions were obtained for the photodissociation of $\mathrm{CH}_{2} \mathrm{O}$ and $\mathrm{CH}_{2} \mathrm{CO} .{ }^{27,28}$ Since then, CO LIF has been used to study the photodissociation of a series of molecules ranging from $\mathrm{CO}_{2}{ }^{29}$ to metal carbonyls. ${ }^{30,31}$ Vacuum-ultraviolet LIF has also been used to obtain $\mathrm{H}_{2}(v, J)$ distributions from the photodissociation of $\mathrm{H}_{2} \mathrm{CO}^{32}$

Virtually all photodissociation/LIF experiments that have been performed subsequent to the examples given here involve the detection of one (or more) of the photofragments mentioned above; the vast number of such experiments obviously precludes mentioning all of them here. In recent years, there has been some development of new detection schemes for photofragments. For example, LIF detection has been implemented for $\mathrm{NH}\left(\mathrm{X}^{3} \Sigma^{-}\right)$, enabling the study of both singlet and triplet $\mathrm{NH}$ from $\mathrm{HN}_{3}{ }^{33}$ and HNF. ${ }^{34}$ The photodissociation of propionaldehyde $\left(\mathrm{CH}_{3} \mathrm{CH}_{2} \mathrm{CHO}\right)$ has been investigated through rotationally resolved LIF of the HCO product. ${ }^{35}$

However, since the mid-1980s, much of the interest in this class of experiments has focused on using LIF to learn not just about product state distributions, but about the various types of photofragment alignment that occur in photodissociation. The earliest photodissociation experiments showed that the velocities of the photofragments are, in general, anisotropic with respect to the polarization vector $\mathbf{E}$ of the photodissociating light source $(\mathbf{v} \cdot \mathbf{E}$ correlation). A more subtle effect is the anisotropy of the photofragment rotational motion with respect to the laser polarization $(\mathbf{J} \cdot \mathbf{E}$ correlation). This latter effect was first seen in (non-rotationally resolved) emission from electronically excited photofragments produced by the vacuum photodissociation of $\mathrm{H}_{2} \mathrm{O}, \mathrm{HCN}$, and $\mathrm{BrCN}^{36}$ In these experiments, the emission is polarized with respect to $\mathbf{E}$, thus probing whether the transition dipole is parallel or perpendicular to the plane of the molecule. An elegant theoretical treatment showed that it is necessary to consider this anisotropy in order to extract accurate rotational distributions from emission and laser-induced fluorescence experiments. ${ }^{37}$ The $\mathbf{J} \cdot \mathbf{E}$ correlation was seen for the first time for individual photofragment rotational states in LIF studies of $\mathrm{H}_{2} \mathrm{O}^{22}$ and $\mathrm{HONO}^{24}$ photodissociation. This effect is observed by measuring the relative intensities of the photofragment rotational branches for probe laser polarizations parallel and perpendicular to that of the photolysis laser.

One obtains yet another level of information on photofragment alignment when LIF of individual photofragment rotational states is combined with Doppler spectroscopy. This is discussed in section II.C.

2. Multiphoton Ionization. The investigation of photofragment state distributions using resonantly enhanced multiphoton ionization (REMPI) offers several advantages, in principle, over LIF detection. REMPI is inherently mass-selective, thereby eliminating any uncertainty concerning the identity of the photofragment. In addition, since a large fraction of the ions produced by the probe pulse are collected in a typical experiment, the sensitivity of this method is extremely high. Finally, REMPI can be used to study photofragments which do not have a high quantum yield for fluorescence. However, even though the first investigation ${ }^{38}$ of photodissociation using REMPI on the photofragments was conducted just shortly after the first photodissociation/LIF experiments, there have been far fewer studies using REMPI as the probe technique. One can trace this to the inherent nonlinearity of the REMPI detection scheme. For most molecules, REMPI involves a two-photon transition between the initial and excited photofragment electronic states, and the extraction of accurate populations from such a measurement requires considerable care. Power broadening from the intense laser fields needed to drive multiphoton transitions can affect the measured intensities, as can electronic states lying between the initial and excited states. Nonetheless, REMPI has 
proved to be a very powerful technique in state-resolved photodissociation studies.

In the first example of REMPI applied to photodissociation, the $\mathrm{NO}$ fragment from $\mathrm{NO}_{2}$ photolysis was detected in a onecolor experiment in which $\mathrm{NO}_{2}$ was photolyzed around $382 \mathrm{~nm}$, and the NO fragment was ionized by a $2+1$ process through the $\mathrm{C}^{2} \Pi$ state. ${ }^{38}$ However, definitive NO populations via REMPI were not reported until several years later. ${ }^{39,40}$ A notable success of the REMPI scheme has been the extraction of rotational and vibrational populations of the $\mathrm{CH}_{3}$ and $\mathrm{CD}_{3}$ fragments produced from the photodissociation of $\mathrm{CH}_{3} \mathrm{I}$ (and $\left.\mathrm{CD}_{3} \mathrm{I}\right) .{ }^{41-43}$ Among other things, these studies demonstrate the importance of reactant initial conditions on the photofragments. In ref 41 , an effusive (thermal) source of $\mathrm{CD}_{3} \mathrm{I}$ was used, and the resulting $\mathrm{CD}_{3}$ rotational distribution showed a propensity for levels with $N=K$. In contrast, a supersonic beam was used in the experiments of ref 42 , and the rotational population of the methyl fragment was concentrated in low $K$ levels. Thus, there appears to be relatively little rotational excitation about the $\mathrm{CH}_{3}$ symmetry axis in $\mathrm{CH}_{3} \mathrm{I}$ photodissociation; $K$ is approximately conserved and reflects the initial rotational population in the $\mathrm{CH}_{3} \mathrm{I}$. This propensity has been further confirmed in a recent study in which rotationally state selected and oriented $\mathrm{CD}_{3} \mathrm{I}$ was photolyzed and the $\mathrm{CD}_{3}$ product characterized by REMPI. ${ }^{44}$

Two REMPI schemes have been developed to probe $\mathrm{H}_{2}$ as a photofragment. One of these is a $2+1$ ionization scheme in which the double-minimum E,F state of $\mathrm{H}_{2}$ serves as the intermediate state; this state lies about $12 \mathrm{eV}$ above the ground state. REMPI via this state is now sufficiently well calibrated ${ }^{45}$ to enable one to extract ground state populations, a nontrivial enterprise given the complicated level structure of the E,F state. Using this scheme, $\mathrm{H}_{2}$ was determined to be a primary product of $\mathrm{H}_{2} \mathrm{~S}$ photodissociation, ${ }^{46}$ and the $\mathrm{H}_{2}(v, J)$ distribution from the $193 \mathrm{~nm}$ photodissociation of mono- and dichloroethylenes was measured. ${ }^{47}$ The other $\mathrm{H}_{2}$ detection scheme involves $1+$ 1 ionization through the $\mathrm{B}$ or $\mathrm{C}$ state using a vacuum-ultraviolet laser operating in the $10-12 \mathrm{eV}$ range. Although the laser system is more complicated, the extraction of populations is easier as this scheme involves one-photon excitation through a simpler intermediate state. Using this scheme, $\mathrm{H}_{2}$ distributions from the photodissociation of cyclohexadiene ${ }^{48}$ and 1,3-butadiene $^{49}$ have been reported.

Additional examples of photodissociation/REMPI experiments include a study of $\mathrm{HN}_{3}$ photodissociation, ${ }^{50}$ in which both the $\mathrm{NH}\left(\mathrm{a}^{1} \Delta\right)$ and $\mathrm{N}_{2}$ photofragments were probed by MPI, and a study of $\mathrm{HCl}$ elimination from vinyl chloride. ${ }^{51}$ However, as with photodissociation/LIF measurements, much of the interest in using REMPI to probe photodissociation stems from one's ability to extract not just rotational state information, but also velocity and alignment information for individual photofragment rotational states. This will be discussed in section II.C.

3. Other State-Resolved Techniques. In order to use either LIF or REMPI in a photodissociation experiment, one of the products must have an optically accessible excited electronic state that is long-lived with respect to predissociation. Thus, when using REMPI in methyl iodide photodissociation experiments, the predissociation rate of the $\mathrm{CH}_{3}$ Rydberg intermediate state is sufficiently fast so that full rotational resolution is not achieved; this is less of a problem for the more slowly predissociating $\mathrm{CD}_{3}$ state, which is why several photodissociation/REMPI experiments have been performed on $\mathrm{CD}_{3} \mathrm{I} .{ }^{41,42}$ The simplest solution to this situation, in principle, is to use direct infrared absorption to probe the product state distribution. This has been done for the $\mathrm{CH}_{3}$ fragment from $\mathrm{CH}_{3} \mathrm{I}$ photodissocia- tion. ${ }^{52,53}$ The lower sensitivity of this technique compared to LIF or REMPI means relatively high densities are required, however, and the $\mathrm{CH}_{3}$ rotational distributions reported in these studies are clearly affected by collisional energy transfer (although the vibrational populations appear to be nascent).

Another probe which does not require a suitable excited state is coherent anti-Stokes Raman spectroscopy (CARS). In a photodissociation/CARS experiment, the photofragments interact with two laser beams at frequencies $\omega_{\mathrm{p}}$ and $\omega_{\mathrm{s}}$, and when $\omega_{\mathrm{p}}-\omega_{\mathrm{s}}$ is resonant with a Raman transition, CARS signal is observed at $\omega_{\mathrm{as}}=2 \omega_{\mathrm{p}}-\omega_{\mathrm{s}}$. In a series of such measurements, the vibration-rotation distribution of $\mathrm{O}_{2}$ produced by photodissociation of $\mathrm{O}_{3}$ in the Chappuis ${ }^{54}$ and Hartley ${ }^{55}$ bands was determined. The rotational distributions were centered at high $J$ but were very narrow, indicating that the $\mathrm{O}_{3}$ dissociation dynamics could be explained with a relatively simple impulsive model. The $\mathrm{H}_{2}(v, J)$ distribution from $\mathrm{H}_{2} \mathrm{CO}$ photodissociation has been measured by CARS, although not under collision-free conditions. ${ }^{56}$ CARS was also used to probe the $\mathrm{CH}_{3}$ fragment from photodissociation of $\mathrm{CH}_{3} \mathrm{I}$ in a free jet. ${ }^{57}$ Nascent vibrational distributions and "near-nascent" (3-6 collisions) rotational distributions were reported; these distributions reflected the propensity toward low- $K$ products seen in the earlier (collision-free) REMPI study. ${ }^{42}$

Time-resolved Fourier transform emission spectroscopy provides another "vibrationally based" photofragment detection scheme, although it is only sensitive to vibrationally and/or electronically excited products. In these experiments, a photolysis (excimer) laser is synchronized with a Fourier transform infrared spectometer in such a way that an interferogram of the emitting photodissociation products is obtained. ${ }^{58}$ The resulting Fourier transform is of sufficient resolution to resolve rotational structure, and the sensitivity of the technique enables one to run at sufficiently low pressure so that little or no rotational relaxation occurs. This method has been used to obtain vibration-rotation distributions of $\mathrm{CO}$ from acetone photodissociation $^{59}$ and $\mathrm{HF}$ from $1,1-\mathrm{CH}_{2} \mathrm{ClF}$. ${ }^{60}$ One can also obtain rotational distributions of low-lying excited states produced by photodissociation that emit in the near-infrared; examples include $\mathrm{CCH}\left(\mathrm{A}^{2} \Pi\right)$ from the photolysis of $\mathrm{C}_{2} \mathrm{H}_{2}{ }^{61}$ and $\mathrm{NH}_{2}\left(\mathrm{~A}^{2} \mathrm{~A}_{1}\right)$ from $\mathrm{NH}_{3}{ }^{62}$

4. Doppler Spectroscopy. Doppler spectroscopy of atomic and molecular photofragments has become a very powerful tool in studies of photodissociation dynamics. In these experiments, one typically scans across an atomic or molecular transition with a tunable laser and monitors the fluorescence or ionization signal as a function of laser frequency. For atomic photofragments, the resulting profile is sensitive to the projection of the photofragment velocity vector onto the propagation direction of the probe laser and contains information on the speed and angular distribution of the photofragments. For molecular photofragments, one also obtains information on the alignment of the product angular momentum. ${ }^{63,64}$ Molecular Doppler spectroscopy is considered in more detail in section II.C.

The appeal of atomic Doppler spectroscopy in photodissociation is that, with sufficiently high translational energy resolution, one could in principle determine the internal energy distribution of the other (atomic or molecular photofragment). For a given kinetic energy release, the Doppler profile for $\mathrm{H}$ atoms will cover the widest frequency spread, so $\mathrm{H}$ atom detection should give the highest energy resolution. Doppler spectroscopy on $\mathrm{H}$ atoms was first demonstrated in the photodissociation of $\mathrm{HI}$, in which the contributions from the $\mathrm{H}+$ $\mathrm{I}\left({ }^{2} \mathrm{P}_{3 / 2}\right)$ and $\mathrm{H}+\mathrm{I}^{*}\left({ }^{2} \mathrm{P}_{1 / 2}\right)$ channels could be distinguished. ${ }^{65}$ This experiment required the use of a tunable VUV laser to map out 
the Doppler profile of the Lyman- $\alpha$ transition. A variation on this technique, velocity-aligned Doppler spectroscopy (VADS), provides significantly higher energy resolution by introducing a delay between the photolysis and probe pulses. ${ }^{66}$ This reduces the contribution of those fragments scattered perpendicular to the probe direction, for which the Doppler shift is very small, and results in cleaner Doppler profiles. However, as demonstrated for $\mathrm{H}_{2} \mathrm{~S}$ photodissociation, ${ }^{66 \mathrm{~b}} \mathrm{VADS}$ provides at best only vibrational resolution for the undetected photofragment. Its resolution is therefore considerably lower than the $\mathrm{H}$ atom timeof-flight experiments discussed in section II.A. Atomic Doppler spectroscopy in which $\mathrm{H}$ atoms as well as heavier species are detected has been used to probe the photodissociation dynamics of several polyatomic species, ${ }^{67-69}$ but these studies typically yield broad translational energy distributions with no resolved structure corresponding to the undetected photofragment.

C. Correlated Product Measurements in Photodissociation. Much of the above discussion has centered on the measurement of product state distributions for a single photofragment. This is sufficient to completely describe product energy disposal in a relatively small number of circumstances, namely, when the other fragment is an atomic species in a single electronic state. The next level of detail, and one that has occupied a central role in more recent photodissociation studies, involves the measurement of correlated product attributes such as the velocity distribution of a photofragment in a specific $(v, J)$ state. Correlated measurements also provide information on photofragment alignment that cannot be determined by measurement of LIF or REMPI intensities alone. In this section we attempt to describe and classify the myriad of correlated measurements that has been used to investigate photodissociation dynamics.

1. LIF/Doppler Studies. The most common correlated measurement in photodissociation involves measuring the Doppler profiles for individual LIF transitions of molecular photofragments. This yields the velocity distribution of the photofragment, and since the internal energy of this fragment is precisely specified through the selected LIF transition, one can infer the internal energy distribution of the other fragment. The degree to which this can be done depends on the initial velocity spread of reactant and the resolution of the probe laser, but in general one obtains average rather than specific energy distributions in the undetected photofragment.

In early studies of this type, Doppler broadening was reported in the LIF of $\mathrm{OH}$ fragments produced from $\mathrm{H}_{2} \mathrm{O}_{2}$ photodissociation, ${ }^{23}$ and Doppler profiles were more systematically examined for the $\mathrm{OH}$ product from $\mathrm{HONO}$ photodissociation ${ }^{24}$ and for $\mathrm{NO}$ from $\left(\mathrm{CH}_{3}\right)_{2} \mathrm{NNO}$ dissociation. ${ }^{70}$ In a study of ICN photodissociation, Doppler spectroscopy on selected CN states was used to determine the correlation between $\mathrm{CN}$ rotation and the electronic state of the I atom; ${ }^{71}$ it was found that low- $J$ states were predominantly from the I* $\left.{ }^{2} \mathrm{P}_{1 / 2}\right)$ channel, whereas higher- $J$ states were correlated with ground state $\mathrm{I}\left({ }^{2} \mathrm{P}_{3 / 2}\right)$. It is generally difficult to obtain more specific information than this without some prior knowledge of the dissociation dynamics. For example, $\mathrm{H}_{2} \mathrm{CO}$ is known to dissociate mainly to $\mathrm{CO}(v=0)$, so from Doppler profiles of the $\mathrm{H}_{2}$ fragment one could determine the correlation between $\mathrm{H}_{2}(v, J)$ levels and average $\mathrm{CO}(v=0)$ rotational excitation, demonstrating that the latter decreases with increasing vibrational excitation of the $\mathrm{H}_{2} \cdot{ }^{32}$

While the energy resolution provided by Doppler spectroscopy is somewhat limited, the real power of Doppler/LIF measurements was demonstrated in a series of experimental ${ }^{72-75}$ and theoretical ${ }^{76}$ papers published in 1986 . These studies showed that the shapes of Doppler profiles are sensitive to the photofragment alignment. As discussed in section II.A, the intensities of LIF transitions and their dependence on polarization yields the $\mathbf{J} \cdot \mathbf{E}$ vector correlation, i.e., the alignment of product rotation with respect to the space-fixed direction defined by the photolysis laser polarization. However, from the Doppler profile shapes, one can extract the vector correlation between product rotation and recoil velocity, the $\mathbf{J} \cdot \mathbf{v}$ correlation. This correlation is of considerable interest because its magnitude is independent of the lifetime of the dissociating state; this is in contrast to the $\mathbf{v} \cdot \mathbf{E}$ and $\mathbf{J} \cdot \mathbf{E}$ correlations, which are at least partially averaged out if the predissociation lifetime is greater than a rotational period. Moreover, the $\mathbf{J} \cdot \mathbf{v}$ correlation yields significant insight into quite complex photodissociation processes in which two molecular fragments are produced.

In order to extract the $\mathbf{J} \cdot \mathbf{v}$ correlation, one measures Doppler profiles for two transitions originating from the same $J$ level, usually a Q-branch and a P- or R-branch transition. This is typically done for at least two polarization and propagation configurations for the photolysis and probe lasers (coaxial propagation with parallel and perpendicular polarizations, for example). Doppler profiles are also sensitive to the photofragment angular distribution, so this must be accounted for in the analysis. The complete treatment of the effect of various correlations on Doppler profiles is given in ref 76, and a more qualitative discussion is offered in a series of review articles. ${ }^{63,64,77}$ Figure 2 shows Doppler profiles of various rotational transtions for the $\mathrm{CO}$ product from OCS photodissociation; for a given rotational state, one can see the dramatic variations of peak profile depending on the laser polarization and whether an R- or Q-branch transition is probed. ${ }^{78}$

As examples, studies of the NO fragment from the dissociation of $\left(\mathrm{CH}_{3}\right)_{2} \mathrm{NNO}^{72}$ and $\mathrm{CH}_{3} \mathrm{ONO}^{79}$ indicate a preference for $\mathbf{J} \perp \mathbf{v}$, supporting a dissociation mechanism in which the NO rotation results from impulsive energy release from the dissociating $\mathrm{N}-\mathrm{N}$ and $\mathrm{N}-\mathrm{O}$ bonds. A similar observation for $\mathrm{CO}$ produced from glyoxal $(\mathrm{CHOCHO})$ photodissociation showed the dissociation occurs in the original plane of the molecule. ${ }^{80}$ In contrast, for $\mathrm{H}_{2} \mathrm{O}_{2}$ photodissociation, one finds that $\mathbf{J}$ and $\mathbf{v}$ tend to be parallel in the $\mathrm{OH}$ fragment, ${ }^{74,75,81}$ indicating that $\mathrm{OH}$ rotation results from torsional motion in the dissociating $\mathrm{H}_{2} \mathrm{O}_{2}$ state. A similar effect is seen in the $\mathrm{NH}\left({ }^{1} \Delta\right)$ fragment from $\mathrm{HN}_{3}$ photodissociation. ${ }^{82}$ More recently, the $\mathbf{J} \cdot \mathbf{v}$ and other vector correlations have been obtained from a variety of LIF/ Doppler measurements, including $\mathrm{H}_{2}$ from $\mathrm{H}_{2} \mathrm{CO}$ photodissociation, ${ }^{83} \mathrm{OH}$ from $\mathrm{CH}_{3} \mathrm{OOH}^{84}$ and $\mathrm{HONO}_{2},{ }^{85}$ triplet and singlet $\mathrm{NH}$ from $\mathrm{HN}_{3},{ }^{33} \mathrm{CN}$ from $\mathrm{CH}_{3} \mathrm{SCN}$ and $\mathrm{NCCN},{ }^{86}$ and $\mathrm{NO}$ from a series of nitrites ${ }^{87}$ and from $\mathrm{CH}_{3} \mathrm{CCl}(\mathrm{NO}) \mathrm{CH}_{3} .{ }^{88}$

2. Correlated REMPI Measurements. One can also obtain velocity and alignment information for state-selected photofragments in REMPI experiments. One way to do this is to measure Doppler profiles for rotationally resolved REMPI transitions, as was done for the $\mathrm{H}_{2}$ fragment from chloroethylene photodissociation. ${ }^{47}$ However, REMPI experiments offer an advantage over LIF studies because one can extract state-specific photofragment velocity and angular distributions without using a high-resolution laser to scan over the Doppler profile for each product state. This can be done by measuring the ion time-offlight distribution produced from a REMPI transition or by measuring the two-dimensional spatial distribution for stateselected photofragments, a technique known as photofragment imaging.

The use of ion time-of-flight in correlated photodissociation measurements was first demonstrated in studies of $\mathrm{NO}_{2}{ }^{39,89}$ and $\mathrm{CD}_{3} \mathrm{I}^{41,90}$ photodissociation. In these experiments, the parent species is dissociated, the $\mathrm{NO}$ or $\mathrm{CD}_{3}$ fragment is state- 

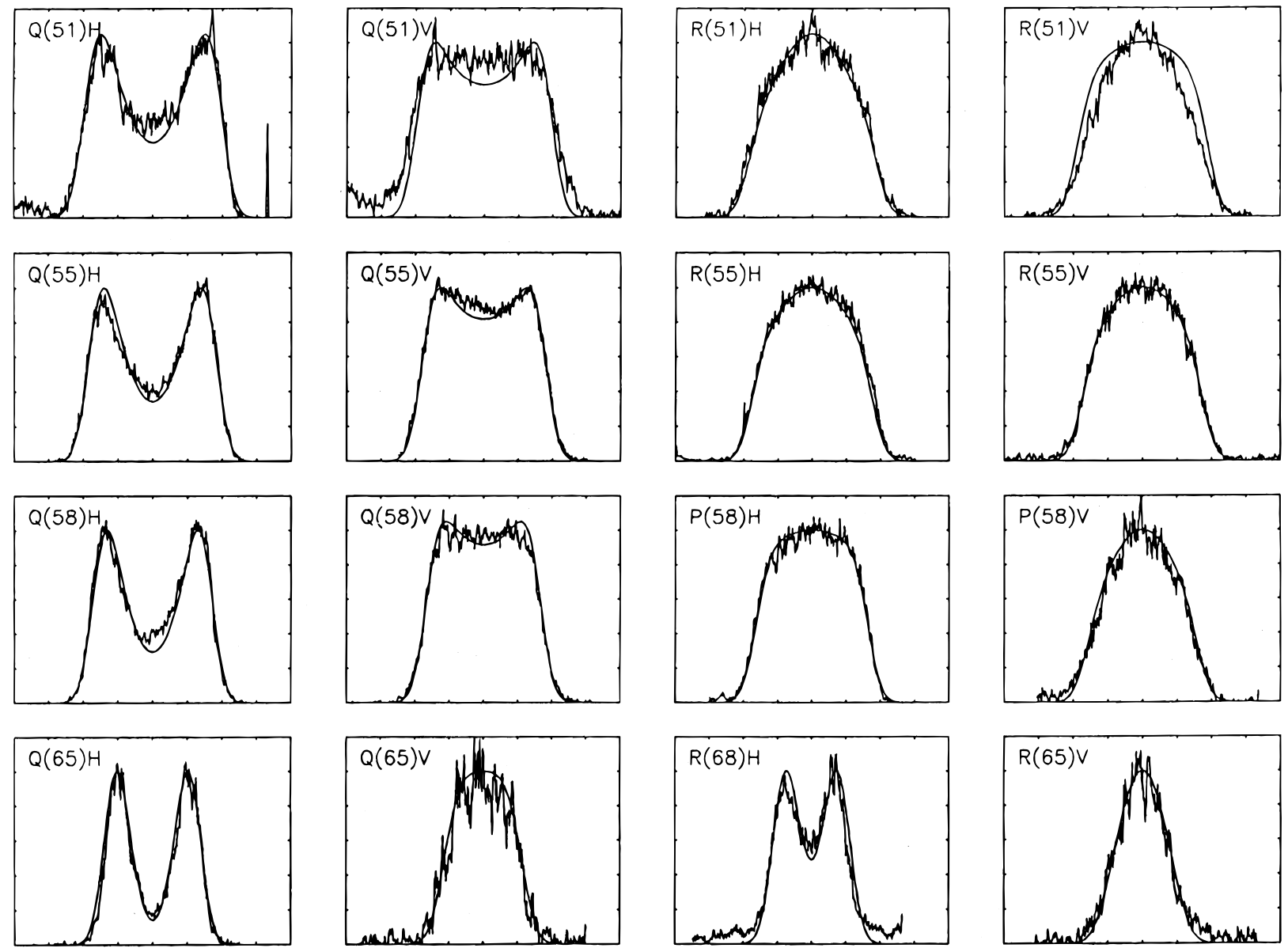

Figure 2. Doppler profiles of representative $\mathrm{CO}(J)$ lines from OCS photodissociation. $\mathrm{Q}(J), \mathrm{P}(J)$, and $\mathrm{R}(J)$ represent different rotational transitions, whereas $\mathrm{H}$ and $\mathrm{V}$ refer to the polarization angle $\theta$ between the probe and photolysis lasers; $\theta=0^{\circ}$ for $\mathrm{H}$ and $90^{\circ}$ for $\mathrm{V}$. Reproduced with permission from ref 78. Copyright 1988 American Institute of Physics.

specifically ionized via REMPI, and the ions are detected in a time-of-flight mass spectrometer. The velocity distribution of the fragments along the axis of the mass spectrometer is reflected in the arrival time distribution of the ionized species. The arrival time distribution represents a one-dimensional projection of the photofragment velocity and angular distribution, analogous to the Doppler profile obtained in a LIF experiment and with comparable or higher resolution. In the $\mathrm{CD}_{3} \mathrm{I}$ studies, for example, one could clearly determine the $\mathrm{I} / \mathrm{I}^{*}$ ratio as a function of $\mathrm{CD}_{3}$ internal state. The $\mathrm{NO}_{2}$ work indicated a propensity for $\mathrm{NO}(v=1, J)$ levels to be correlated with the $\mathrm{O}$ atoms in their ground ${ }^{3} \mathrm{P}_{2}$ spin-orbit level. The shapes of the arrival time distributions as a function of the various laser polarization angles ${ }^{91}$ can also be used to extract information on photofragment alignment, again in analogy to Doppler measurements. Examples of more recent work incorporating ion time-of-flight include studies of $\mathrm{HN}_{3}{ }^{34}$ and acetone ${ }^{92}$ photodissociation.

The technique of photofragment imaging ${ }^{93,94}$ provides an elegant means of obtaining velocity and angular distributions for state-selected products. In these experiments, parent species are photodissociated, and photofragments are state-specifically ionized via REMPI immediately after photolysis. The resulting ion packet is then accelerated toward a position-sensing detector which yields a two-dimensional image of the ions. During the transit time to the detector, the ions separate spatially according to the speed and angular distribution of the photofragments, and this is reflected in the two-dimensional image. A significant advantage of this technique over the "one-dimensional" Doppler and ion time-of-flight methods is that, under conditions of cylindrical symmetry, the two-dimensional image can be directly inverted to give the three-dimensional velocity distribution of the state-selected photofragments. ${ }^{42}$ Photofragment imaging has been used to obtain rotationally state selective velocity distributions for the dissociation of $\mathrm{CH}_{3} \mathrm{I}$ and $\mathrm{CD}_{3} \mathrm{I},{ }^{42} \mathrm{NO}_{2},{ }^{40} \mathrm{O}_{3},{ }^{95}$ and $\mathrm{CH}_{3} \mathrm{COCl}^{96}$ One can also perform somewhat less detailed experiments in which atomic photofragments are state-specifically ionized and analyzed via photofragment imaging. ${ }^{97-101}$ The results of ref 100 are of particular interest as they provided the first evidence for the production of highly vibrationally excited $\mathrm{O}_{2}\left({ }^{3} \Sigma_{\mathrm{g}}{ }^{-}\right)$from $\mathrm{O}_{3}$ photolysis; this product may be partially responsible for stratospheric $\mathrm{O}_{3}$ production.

Information on photofragment alignment can be extracted from imaging experiments, although this has so far only been successful for $\mathrm{CD}_{3} \mathrm{I}^{102}$ and $\mathrm{O}_{3} .{ }^{103}$ In a particularly impressive recent experiment, photofragment imaging was used to determine the angular distribution from photodissociation of $\mathrm{CD}_{3} \mathrm{I}$ which was spatially oriented by means of a hexapole field; ${ }^{104}$ the resulting asymmetric image reflected the anisotropic initially selected $\mathrm{CD}_{3} \mathrm{I}$ symmetric top wave function.

3. Other Correlated Measurements. Very recently, two new methods for obtaining correlated state distributions from photodissociation have been reported. The first of these yielded explicit and detailed correlations between NO internal states and the spin-orbit levels of the $\mathrm{O}^{3} \mathrm{P}$ atom from $\mathrm{NO}_{2}$ photodissociation; the experimental arrangement is shown in Figure 3. ${ }^{105} \mathrm{NO}_{2}$ in a free jet was photolyzed near the dissociation threshold, and the $\mathrm{NO}+\mathrm{O}$ products were allowed to travel downstream for several microseconds prior to multiphoton ionization of the NO product. During this interval, NO in a given internal state that is correlated with an $\mathrm{O}$ atom in its ground 


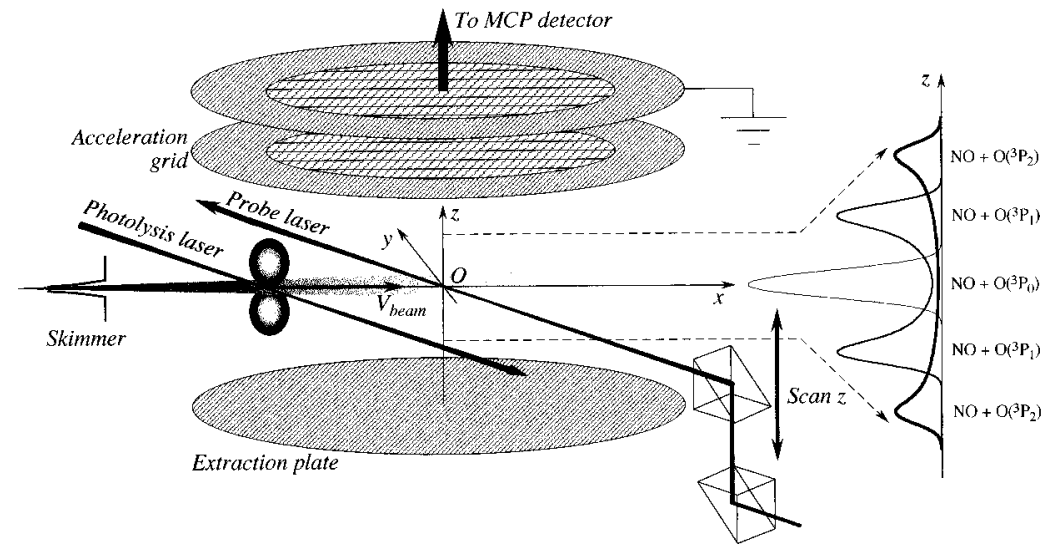

Figure 3. Experimental arrangement for correlated $\mathrm{NO}_{2}$ photodissociation study in ref 105 . Shown on the right are examples of the broadened single-mode distributions of $\mathrm{NO}$ correlated with different states of $\mathrm{O}\left({ }^{3} \mathrm{P}\right)$ calculated assuming ${ }^{3} \mathrm{P}_{2}:{ }^{3} \mathrm{P}_{1}:{ }^{3} \mathrm{P}_{0}=1: 1: 0.1$. The $\mathrm{O}\left({ }^{3} \mathrm{P}_{2}\right), \mathrm{O}\left({ }^{3} \mathrm{P}_{1}\right)$, and $\mathrm{O}\left({ }^{3} \mathrm{P}_{0}\right)$ channels have 227,69, and $1 \mathrm{~cm}^{-1}$ available as relative translational energies, respectively. A recoil anisotropy parameter of $\beta=1.35$ was used here. Reproduced with permission from ref 105 .

${ }^{3} \mathrm{P}_{2}$ state will move further off the beam axis than if the $\mathrm{O}$ atom were in the ${ }^{3} \mathrm{P}_{1}$ or ${ }^{3} \mathrm{P}_{0}$ levels (the photolysis laser polarization is perpendicular to the beam axis, and $\beta \approx 1.4$ for this transition), so by moving the probe beam perpendicularly to the beam axis, one can selectively ionize $\mathrm{NO}$ correlated with a specific $\mathrm{O}$ atom state. This experiment shows marked fluctuations of the $\mathrm{O}$ atom distribution as a function of NO internal state; in some cases, the $\mathrm{O}$ atom distributions for the two $\Lambda$-doublet components of the same NO rotational level are different.

In the second method, $\mathrm{CO}$ photofragments in a selected $(v, J)$ level are excited to the metastable $\mathrm{a}^{3} \Pi$ state; molecules in this metastable state can be detected with very high efficiency using a particle multiplier. By measuring the time-of-flight distribution of the metastable $\mathrm{CO}$ at a particular scattering angle, one can determine the internal energy of the other fragment through energy balance. In a study of ketene photolysis, ${ }^{106}$ the resolution was sufficient to determine the vibrational distribution of the $\mathrm{CH}_{2}$ fragment associated with a particular $\mathrm{CO}(v, J)$ state. This method holds considerable promise for the numerous photodissociation processes in which $\mathrm{CO}$ is generated.

D. New Techniques. 1. Resonant Four-Wave Mixing Processes. Recent experiments have demonstrated that resonant four-wave mixing provides an elegant means to study the spectroscopy ${ }^{107,108}$ and photodissociation dynamics ${ }^{109,110}$ of molecules. In a study of $\mathrm{NO}_{2}$ photodissociation in a free jet, ${ }^{109}$ two laser beams at the same frequency cross at a small angle $\theta$ inside the jet. Interference between the beams generates a spatially varying electric field, and by choosing the laser frequency to be one at which $\mathrm{NO}_{2}$ dissociates, one sets up a "population grating" in which the $\mathrm{NO}_{2}$ population is depleted most where the electric field is high, and vice versa. The NO photofragment population also varies spatially, and a probe laser tuned to a specific NO electronic transition will be coherently scattered at an angle determined by the grating (photolysis) and probe laser frequencies and geometries. Immediately after the photolysis pulse, the intensity of the scattered probe laser reflects the population of the selected NO internal state. The subsequent evolution of the scattered probe beam as a function of delay time with respect to the grating pulse depends on the speed and angular distribution of the NO fragment. Near the dissociation threshold, for example, one observes decaying recurrences as the NO population grating disappears and then partially re-forms over a time scale of 100-200 ns.

A somewhat different approach has been used to study $\mathrm{H}_{2} \mathrm{O}_{2}$ photodissociation. ${ }^{110}$ Here, $\mathrm{H}_{2} \mathrm{O}_{2}$ is photodissociated within a gas cell, and the $\mathrm{OH}$ product is detected through a degenerate four-wave mixing scheme. The technique is inherently subDoppler and allows one to extract both product state distributions and alignment information. The general appeal of this class of experments is that the detected photofragment only needs to absorb at the probe wavelength; a high fluorescence yield is not required. On the other hand, due to collective nature of the four-wave mixing process that underlies both experiments, the sensitivity is not as high as in LIF or REMPI experiments.

2. Fast Radical Beam Photodissociation. The vast majority of photodissociation experiments have been performed on stable, closed shell species or relatively unreactive radicals such as $\mathrm{NO}_{2}$. While it might seem straightforward to apply one or more of the above schemes to the photodissociation dynamics of highly reactive radicals, only a few such experiments have been carried out. A major impediment to these experiments is the need for a "clean" source of the radical of interest, so that one can be certain that the detected photofragments come from the radical and not from the radical precursor or other unwanted species.

One approach to this problem has been to generate radicals through laser photodetachment of a fast $(5-8 \mathrm{keV})$ of massselected negative ions. ${ }^{111}$ Photodetachment at a suitable laser frequency produces mass-selected, rotationally cold radicals in their ground vibrational states. The resulting radicals are then photodissociated with a second pulse, and because of the high beam energy, the photofragments are efficiently detected with a multichannel plate detector that lies 1-2 m downstream from the photodissociation region. The detector is configured to detect the two photofragments from a single photodissociation event in coincidence and to yield the positions on the detector face as well as the difference in arrival time for the photofragments. From this, one obtains the masses, kinetic energy release, and scattering angle for each photodissociation event. The resulting translational energy resolution has been demonstrated in a study of $\mathrm{O}_{2}$ photodissociation to be as good as $0.7 \%$, enabling one to determine the correlated spin-orbit distribution of the two $\mathrm{O}^{3} \mathrm{P}_{j}$ atoms from predissociation of the $\mathrm{O}_{2} \mathrm{~B}^{3} \Sigma_{\mathrm{u}}{ }^{-}$ state. ${ }^{112}$ Experiments on $\mathrm{CH}_{3} \mathrm{O}$ yielded the vibrational distribution in the umbrella $\left(v_{2}\right)$ mode of the $\mathrm{CH}_{3}$ fragment at several excitation energies. ${ }^{113}$ Results have also been obtained for the $\mathrm{N}_{3},{ }^{110} \mathrm{NCO},{ }^{114} \mathrm{CH}_{2} \mathrm{NO}_{2},{ }^{115}$ and $\mathrm{CH}_{2} \mathrm{CHO}$ (vinoxy) ${ }^{116}$ radicals. These experiments are, in many cases, the first studies of the photodissociation dynamics of these radicals and represent an expansion of this field to include more electronically complex species than are typically investigated. 


\section{The Application of Photodissociation to Problems Central to Chemical Reaction Dynamics}

A. Section Overview. While excited state spectroscopy and photodissociation dynamics used to be viewed as disjoint from reactive scattering studies of the dynamics of bimolecular ground state reactions, several recent advances rapidly integrated these subfields. First, although the theoretical treatment of photodissociation dynamics had long emphasized the parallel with bimolecular reaction dynamics, ${ }^{117}$ wavepacket theories ${ }^{118}$ established a crucial link between classical reaction dynamics and traditional electronic absorption and emission spectroscopy. Thereafter, many electronic spectroscopy experiments drew heavily on a dynamical perspective to interpret resonant emission spectra and broadened vibrational features in absorption spectra. Second, photodissociation experiments began to play an increasingly important role in developing our understanding of chemical reaction dynamics in general as experimental techniques using lasers and molecular beams advanced ${ }^{119}$ and as the ability to calculate $a b$ initio excited state surfaces grew. Experiments began to use photodissociation to access excited electronic potential energy surfaces in ways not usually accessible to ground state bimolecular reactive scattering experiments. For instance, Franck-Condon excitation allowed access of the saddle point region, or transition state region, of excited electronic potential energy surfaces in several key molecular systems reviewed below. Changing the ultraviolet photon energy or beginning in different vibrational levels in the ground state additionally offered a level of control over the portion of the Franck-Condon region accessed. Finally, several recent experiments have tested and challenged two of the most fundamental assumptions in transition state theories for chemical reactions, the assumptions of intramolecular vibrational energy redistribution, and the separability of electronic and nuclear motion. In the next few sections we detail examples of experiments which illustrate these conceptual advances that link electronic spectroscopy and photodissociation experiments to the study of reaction dynamics in general.

B. A Dynamical View of Electronic Spectroscopies: Wavepackets. Wavepacket theories ${ }^{118,120}$ established a critical link between dynamics and spectroscopy and changed the way we think about electronic absorption and emission spectroscopy, key probes of photodissociation events. While the traditional interpretation of broadened rovibrational features in an electronic absorption spectrum had been, a la Herzberg, ${ }^{121}$ lifetime broadening due to electronic predissociation, Heller ${ }^{118}$ introduced a dynamical perspective to both photoabsorption and photoemission spectroscopy, complementing the traditional timeindependent scattering calculations ${ }^{117,120}$ for photodissociation dynamics. In the wavepacket picture one imagines using a delta function (in time) laser pulse to promote the molecular amplitude from a vibrational level (usually the zero-point level for absorption spectra) in the ground electronic state to the excited electronic state. The excited molecular "wavepacket" undergoes time-dependent dynamics on the excited state potential energy surface. The time-dependent overlap of the moving excited state wavepacket with the initial state gives, when Fourier transformed, the electronic absorption spectrum complete with a dynamical interpretation for the "lifetime broadened" resonances, as shown schematically in Figure 4.

Perhaps the cleanest example of this is in the ultraviolet absorption spectrum of the Ã state of water, which shows diffuse vibrational features in the continuum absorption band. The spectrum is beautifully reproduced by Fourier transforming the wavepacket dynamics propagated on the excited state potential energy surface; the vibrational resonances in the absorption
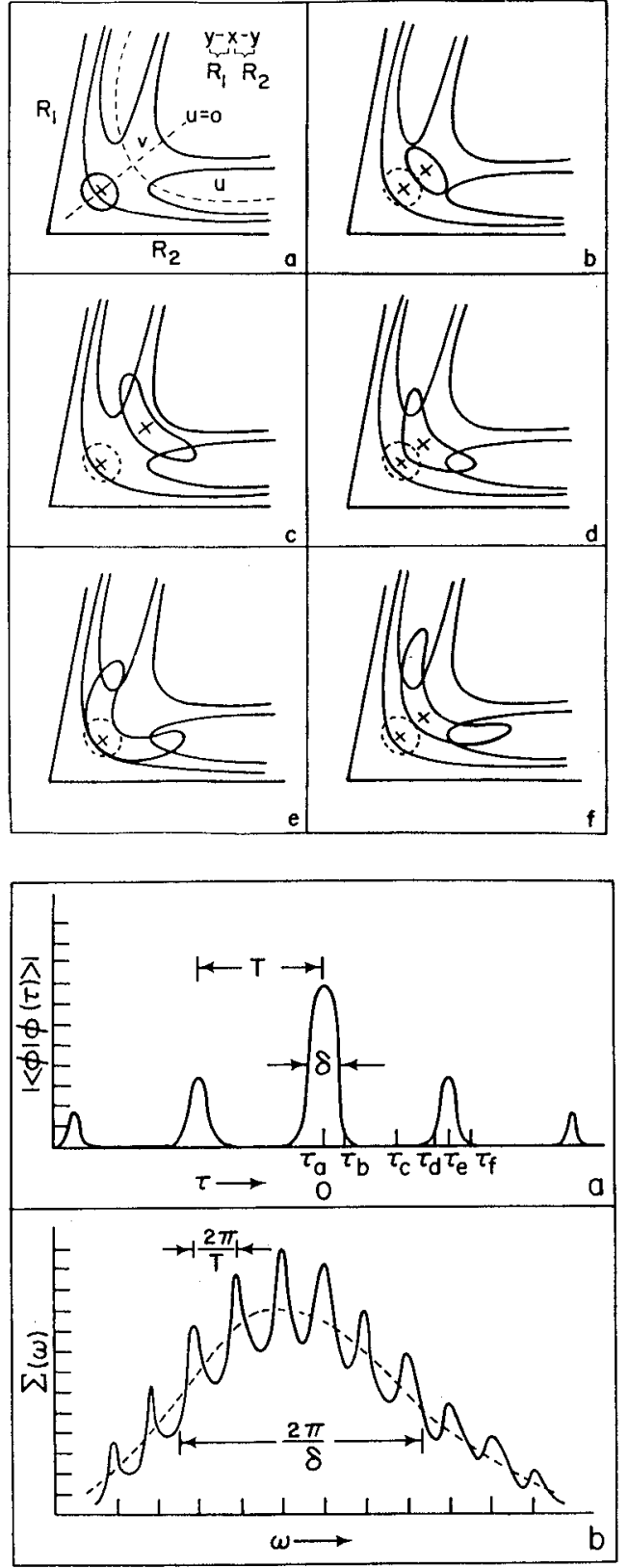

Figure 4. Top frames: Qualitative diagram showing the evolution of a wavepacket $\phi(\tau)$ of a symmetric triatomic molecule on an upper potential energy surface upon excitation with a delta function laser pulse. Bottom frames: (a) The time-dependent overlap of $\phi(0)$ with $\phi$ at later times $\tau$. The symmetric stretch vibrational period is $\mathrm{T}$ in the figure. (b) The Fourier transform of (a), showing the structure that would result in an electronic absorption spectrum. Adapted with permission from Figures 2 and 3 of ref $118 b$.

spectrum result from a small part of the excited amplitude returning to the Franck-Condon region after moving out along the symmetric stretch coordinate on the excited state potential energy surface. ${ }^{122}$ Because only a small fraction of the amplitude makes it back to the Franck-Condon region on the first round trip, and virtually none on the second round trip (forces have driven the amplitude out toward the repulsive regions of the potential energy surface in the $\mathrm{H}+\mathrm{OH}$ exit channels), the Fourier transform gives broadened individual vibrational features, consistent with the traditional interpretation that the lifetime of the vibrational recurrences is short. However, one need not invoke electronic "predissociation"; all the dynamics is occurring on a single Born-Oppenheimer potential energy surface. 
The wavepacket interpretation of spectroscopy has proven extremely useful in a wide variety of experiments, including emission spectroscopy of molecules excited to a dissociative electronic continuum. ${ }^{123}$ In those experiments, the dispersed emission from the dissociating molecules reveals what vibrational levels in the ground electronic state the dissociating molecule developed good Franck-Condon overlap with during dissociation. While early emission spectroscopy experiments focused on direct dissociation on a single potential energy surface, later work probed the dynamics in regions of potential surfaces in which nonadiabatic coupling can induce transitions to other excited potential surfaces, ${ }^{124-127}$ a subject of importance later in this review. Wavepacket dynamics has also been used to model photoelectron detachment spectra that reflect the ground state dynamics of reactive scattering systems like $\mathrm{Br} \cdots \mathrm{H} \cdots \mathrm{I}$ near the saddle point for the $\mathrm{Br}+\mathrm{HI}$ reaction. ${ }^{128}$

C. Accessing the Transition State Regions of Reactive Potential Energy Surfaces Using Photodissociation. Although photodissociation has long been termed a "half-collision" by dynamicists, this concept has deepened and refined as photoexcitation is increasingly used to provide Franck-Condon access to selected regions of reactive potential energy surfaces. Perhaps the most exciting studies are those which directly access the saddle point region, or transition state region, of an excited electronic potential energy surface. For instance, the photoexcitation of $\mathrm{CH}_{3} \mathrm{SH}$ in the first electronic absorption band peaking near $230 \mathrm{~nm}$ promotes the molecule to the $1^{1} \mathrm{~A}^{\prime \prime}$ potential energy surface in the region of the saddle point, or transition state, between the $\mathrm{CH}_{3} \mathrm{~S}+\mathrm{H}$ and $\mathrm{CH}_{3}+\mathrm{SH}$ exit channels on the excited state reaction coordinate. ${ }^{129,130}$ Hydrogen atom Rydberg time-of-flight spectra partially resolve the vibrational population in the $\mathrm{C}-\mathrm{S}$ stretch, ${ }^{131,132}$ and molecular beam photofragment velocity and angular distributions analyze the product branching between the $\mathrm{C}-\mathrm{S}$ and $\mathrm{S}-\mathrm{H}$ exit channels upon excitation to this transition state region. ${ }^{129}$ We return to this system in section III.D.2, as one can also access the transition state region indirectly by exciting to the adiabatically bound $2^{1} \mathrm{~A}^{\prime \prime}$ potential energy surface at higher photon energies, from which nonadiabatic transition to the saddle point region of the $1^{1} \mathrm{~A}^{\prime \prime}$ surface can occur.

Two other photodissociative systems that offer FranckCondon access to the transition state region of an excited state reaction coordinate are $\mathrm{H}_{2} \mathrm{~S}$ and $\mathrm{H}_{2} \mathrm{O}$. They offer the opportunity to spectroscopically observe a vibrational motion, the symmetric stretch, ${ }^{122}$ which is orthogonal to the $\mathrm{OH}(\mathrm{SH})+\mathrm{H}$ $\rightarrow \mathrm{H}+\mathrm{OH}(\mathrm{SH})$ at the transition state, as depicted in Figure 5 for $\mathrm{H}_{2} \mathrm{O} .{ }^{133}$ The emission spectrum of dissociating $\mathrm{H}_{2} \mathrm{~S}^{126,134,135}$ also offers the opportunity to probe the scattering wave function in the $\mathrm{H}+\mathrm{SH}$ exit channel; the emission spectra into high vibrational levels of the ground state show selective overlap with eigenstates with the vibrational quanta localized on one $\mathrm{S}-\mathrm{H}$ oscillator (the one that is breaking) and no bending quanta, reflecting that the scattering dynamics in the exit channel evolves into stretching of one $\mathrm{S}-\mathrm{H}$ bond with no bending and little motion in the other $\mathrm{S}-\mathrm{H}$ bond. This connects beautifully with the asymptotic SH fragment internal state distribution, ${ }^{136}$ which is vibrationally and rotationally cold.

While $\mathrm{H}_{2} \mathrm{~S}$ is complicated by a conical intersection in the Franck-Condon region, the dynamics upon photoexcitation of $\mathrm{H}_{2} \mathrm{O}$ in the first absorption band can be essentially perfectly modeled on a single adiabatic Born-Oppenheimer potential energy surface and affords the opportunity to actively control the bond-breaking dynamics. The fact that the Franck-Condon region is, in this symmetric molecule, centered on the saddle

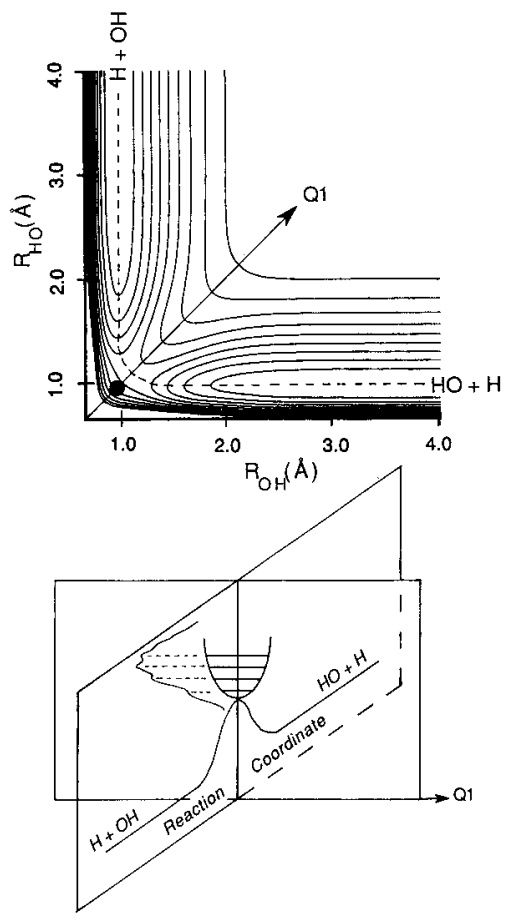

Figure 5. Upper frame shows a contour plot of the first excited electronic potential energy surface for the $\mathrm{H}+\mathrm{OH} \rightarrow \mathrm{HO}+\mathrm{H}$ reaction, the $\tilde{\mathrm{A}}$ state of $\mathrm{H}_{2} \mathrm{O}$ used in ref 122 to model the structure in the ultraviolet absorption spectrum. The black dot near the saddle point represents the Franck-Condon region. The reaction coordinate is shown schematically in dotted line in the upper frame along with the symmetric stretch coordinate Q1. The lower frame shows a schematic diagram of a cut along the reaction coordinate and the resonance energies corresponding to increasing quanta in the symmetric stretch motion Q1 at the saddle point. Recurrences in the symmetric stretch motion result in the structure in the absorption spectrum. Reproduced with permission from ref 133. Copyright 1991 Elsevier.

point means that the excited molecules have equal probability of evolving into the two $\mathrm{O}-\mathrm{H}$ fission exit channels. When one deuterates the $\mathrm{H}_{2} \mathrm{O}$ to make $\mathrm{HOD}$, one finds that $\mathrm{O}-\mathrm{H}$ fission becomes more pronounced than $\mathrm{O}-\mathrm{D}$ fission. ${ }^{137-139}$ This is because the larger zero-point motion in the $\mathrm{O}-\mathrm{H}$ bond in the ground state vibrational wave function channels gives the molecule better Franck-Condon overlap with scattering wave functions on the $\mathrm{O}-\mathrm{H}$ repulsive side of the saddle point than the $\mathrm{O}-\mathrm{D}$ repulsive side. This effect is dramatically enhanced when one photodissociates HOD molecules with one or several quanta of vibrational energy ${ }^{140}$ in the $\mathrm{OH}$ stretch; photodissociation of HOD from the local mode $(\mathrm{mnb})=(400)^{+}$vibrational state in the ground state at $266 \mathrm{~nm}$ results in virtually exclusively $\mathrm{O}-\mathrm{H}$ bond fission, no $\mathrm{O}-\mathrm{D}$ bond fission. Thus, these systems offer a way to induce bond-selective photochemistry by accessing different repulsive regions of one Born-Oppenheimer potential energy surface.

Other kinds of experiments offer the opportunity to probe selected regions of either excited state or ground state reactive scattering potential energy surfaces. For instance, photodissociation experiments using weakly bound clusters permit FranckCondon access to the reactant channel region of a bimolecular reactive collision on an excited electronic potential energy surface. ${ }^{141}$ Photoelectron detachment studies provided analogous opportunities, allowing the spectroscopic study of the transition state region of ground state bimolecular reactions. ${ }^{142}$ In all these studies one sees a unity in the detailed description of molecular dissociation and bimolecular chemical reactions at the molecular quantum level. 
D. Challenges in Photodissociation Dynamics That Impact Reaction Dynamics in General: Elucidating Statistical Assumptions and Nonadiabatic Effects. 1. Gaining a Detailed View of Molecular Dynamics. As our experimental tools to probe photodissociation dynamics have sharpened and our ability to calculate highly accurate excited electronic potential energy surfaces has improved, dynamicists have pursued "exact" quantum scattering calculations to test our ability to predict the dynamics of chemical reactions from first principles. These predictions range in detail from the net rate of traversing any barrier along the dissociation coordinate to a complete set of state-to-state reaction cross sections. For systems where it was a good approximation to assume that the dynamics evolved on a single potential energy surface, the success was stunning, particularly in photodissociation reactions involving only a few atoms. In that case, all the nuclear degrees of freedom could be treated accurately, and given a good $a b$ initio potential energy surface, the scattering calculations gave accurate predictions of product vibrational and rotational states and a detailed picture of the dissociation dynamics. In some cases, experimental observables revealed key features of the reaction dynamics and excited state potential energy surface. For instance, in the photodissociation of $\mathrm{FNO},{ }^{143}$ the NO product rotational state distribution directly reflected the nodes and maxima in the bending wave function of FNO at the barrier, or transition state, on the excited electronic potential energy surface as shown in Figure 6. ${ }^{143,144}$ For larger systems where all the internuclear degrees of freedom could not be included explicitly, workers often drew on statistical models of the reaction dynamics, which assume a statistical distribution of energy among the internal modes (or all but a select set of internal modes) of the dissociating molecule at the transition state. As our ability to predict the dynamics of photodissociation observed in experimental measurements matures, two key assumptions commonly made in those predictions draw increasing scrutiny. One, the assumption in statistical theories of rapid vibrational energy redistribution in the dissociating molecule, has been of interest for decades; ${ }^{145-148}$ we review in section III.D.2 recent work that seeks to understand the transition from mode-selective to statistical behavior. The second assumption, that the dynamics evolves on a single Born-Oppenheimer potential energy surface, is implicit in most statistical theories and often used in exact quantum scattering dynamics calculations without justification. Recent experiments reviewed in section III.D. 3 explore classes of systems in which a breakdown of the BornOppenheimer approximation is the rule rather than the exception.

2. Elucidating the Transition from Mode-Selective to Statistical Behavior with Photodissociation Experiments. Over the past several decades, photodissociation experiments have been used to test one of the foundational assumptions in statistical transition state theories of chemical reactions, that at the transition state of the chemical reaction the vibrational energy in a molecular complex is statistically distributed among all the active modes. ${ }^{146,147,149}$ One way to test the assumption was to photoexcite a molecule via different infrared or vibronic transitions and measure if the resulting photofragment branching ratios, rates, or internal state distributions changed in a modeselective way. Early infrared multiphoton dissociation experiments brought home the fact that the transition state theories require that the distribution of vibrational energy only need be statistical at the transition state. Nothing in the statistical theories precludes exit channel forces (resulting from a barrier to the reverse reaction) from altering this statistical distribution and resulting in asymptotic photofragment kinetic energy or internal state distributions that are highly nonstatistical. ${ }^{145}$ The
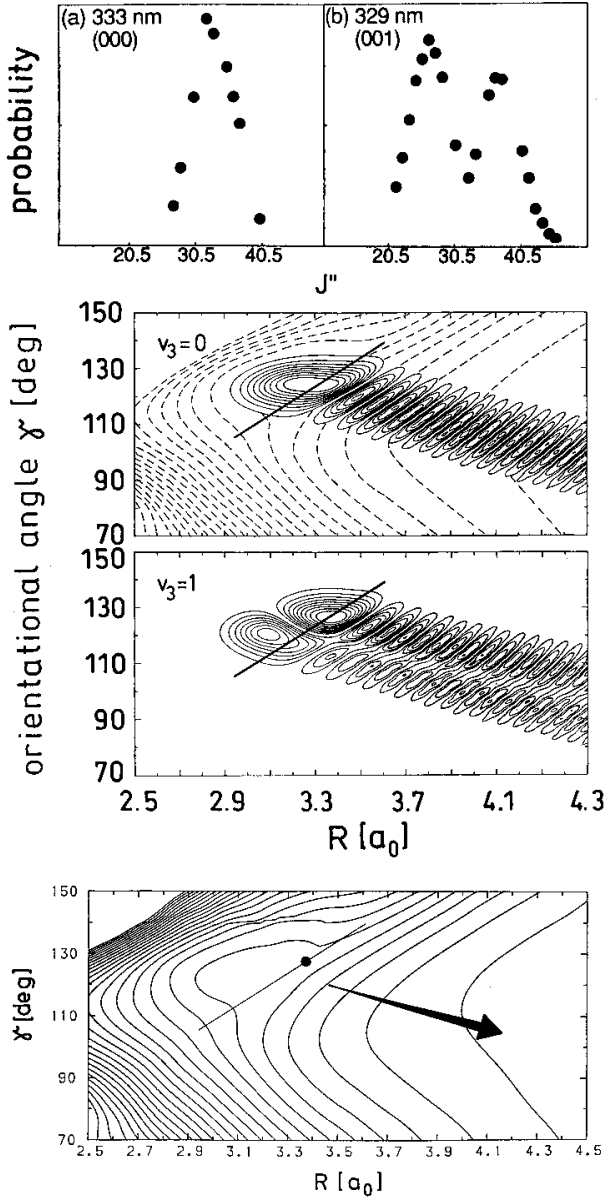

Figure 6. The top part of the figure shows the experimentally measured rotational state distribution of NO following excitation to FNO in a band with $v_{3}=0$ in frame (a) and $v_{3}=1$ in frame (b). The middle two frames in the figure show contour plots of the stationary wave functions $\psi(R, \gamma, E)$ for the two lowest bending resonances supported on the $\mathrm{S}_{1}$ potential energy surface shown in the bottom frame. The ab initio potential energy surface is minimized along $r$ where $R$ is the distance from the $\mathrm{F}$ atom to the center of mass of the NO, $r$ is the NO stretching coordinate, and $\gamma$ is the Jacobi angle. The dot marks the equilibrium geometry in the ground electronic state. Adapted with permission from portions of Figures 2, 4, and 8 of ref 143.

focus of many studies has shifted toward observables that would not be sensitive to exit channel forces. ${ }^{147}$ These studies assessed whether or not the rate of the reaction changed with excitation energy in a monotonic way and whether the branching between energetically allowed fragmentation pathways depended on the vibrational nature of the mode excited. Numerous infrared multiphoton dissociation studies found the product branching ratios were well-predicted by statistical theories. Due to efficient vibrational coupling in strongly bound molecules, one could not selectively cleave a particular molecular bond by tuning the infrared laser into resonance with a particular bond's vibrational frequency.

Although the predissociation rates of weakly bound complexes often showed highly mode-selective behavior, the reaction rates of numerous overtone-initiated photofragmentation or photoisomerization reactions ${ }^{147,149}$ in strongly bound relatively large molecular systems at moderate to high excitation energies remained roughly consistent with statistical theories. Despite this fact, one could still achieve "bond-selective" chemistry simply by photoexciting the molecule to different repulsive electronic states ${ }^{150}$ or different repulsive regions of one excited electronic potential energy surface. ${ }^{140}$ However, these successes did not require the failure of the statistical vibrational energy 
distribution assumption in transition state theories (although the first example requires failure of the assumption of the separability of electronic and nuclear motion ${ }^{151}$ ).

With the qualitative conclusion that mode-selective behavior is commonly seen in strongly bound systems only for small molecules, or for larger molecules only at low excitation energies where the density of states is small, recent work has begun to focus on understanding the transition from modeselective to statistical behavior. Although most of the work in this area involves photodissociation in the ground electronic state, and will be covered in the review by Smith and Moore in this issue, the ability to resonantly excite different vibronic transitions has offered the possibility to investigate modeselective effects in photodissociation experiments involving excited electronic states. Such studies fall into two categories. The first type of study includes photodissociation which, though initiated via a transition whose oscillator strength results from a strong electronic transition, occurs essentially in the ground electronic state via internal conversion; the work reviewed below on $\mathrm{NO}_{2}$ falls into this category. The second type of study occurs on a single Born-Oppenheimer potential energy surface, but an excited state one, so can also directly explore the transition from mode-selective to statistical behavior in the same way that unimolecular dissociation on the ground state potential energy surface can. The highly mode-selective example of FNO described in section III.D.2 is an example of this type of study. Several key examples of both types of photodissociation studies are discussed below, indicating how they deepen our understanding of the statistical assumption invoked in transition state theories.

Many examples of highly mode-selective photodissociation dynamics upon excitation to states with structured electronic absorption spectra are in molecules like FNO. These states are not directly dissociative because their absorption spectra are structured, but they do not sample a very deep well in the potential surface in the Franck-Condon region. A recent review ${ }^{152}$ of vibrational resonances in molecular photodissociation illustrates this point. It analyzes the transition from mode selective to statistical behavior in a series molecules, including $\mathrm{XNO}\left(\mathrm{X}=\mathrm{Cl}, \mathrm{F}, \mathrm{CH}_{3}\right)$ molecules upon ultraviolet photodissociation and $\mathrm{HO}_{2}$ and $\mathrm{NO}_{2}$ molecules upon dissociation on the ground state potential surface. The XNO molecules sample at most a very shallow well on the potential energy surface in the Franck-Condon region and consequently evidence highly mode-selective behavior; the NO product rotational and/or vibrational state distribution is highly sensitive to the nature of the vibrational motion in the vibronic state excited in the absorption spectrum. In contrast, in $\mathrm{NO}_{2}$ and in the model version of $\mathrm{HO}_{2}$ studied, the molecules sample a deep well on the potential surface, namely, the ground electronic state, and evidence statistical dynamics. The review concludes that if the systems sample a deep well and have a strong coupling between internal modes, then the vibrational resonances evidence statistical behavior, with the observables being the widths of the spectral features and the final state distributions of the products measured upon photodissociation at the resonance energy.

Recent experiments ${ }^{105,153}$ on $\mathrm{NO}_{2}$ have investigated how the molecular dissociation, while behaving statistically on the average, exhibits sometimes dramatic fluctuations about the average when state-resolved experiments are done. Using both single-photon and infrared-visible excitation, the experiments measured the internal state distributions of the $\mathrm{NO}$ product upon state-selective laser excitation of the $\mathrm{NO}_{2}$ and the partial absorption spectra for $\mathrm{NO}_{2}$ upon detecting one $\mathrm{NO}$ quantum state. The investigators conclude that although $\mathrm{NO}_{2}$ decomposi-
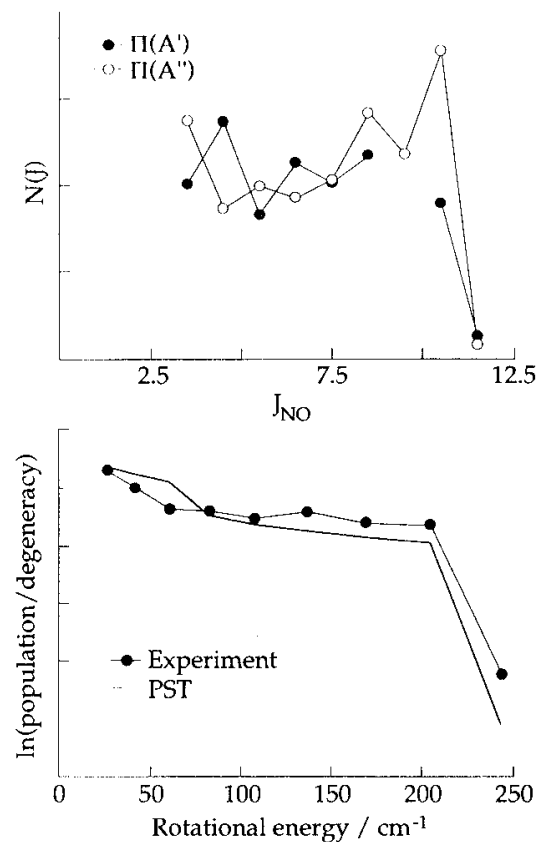

Figure 7. $\mathrm{NO}\left({ }^{2} \mathrm{P}_{1 / 2}\right)$ rotational state distributions from $\mathrm{NO}_{2}$ excited via IR-visible double-resonance excitation to an energy $235 \mathrm{~cm}^{-1}$ above the dissociation limit. The upper panel displays distributions in the two $\Lambda$-doublet states of $\mathrm{NO}$; the lower panel compares in a Boltzmann plot the distribution summed over $\Lambda$-doublet levels with the predictions of phase space theory. Reproduced with permission from ref 153e. Copyright 1994 American Institute of Physics.

tion satisfies the fundamental assumptions of statistical theories, where the initial states are ergodic and the formation and decay of the excited complex take place on different time scales, the density of states and the number of final states are small enough that effects arising from the decay of resonances can be observed. Under state-to-state conditions the averaging inherent in statistical theories is removed, and effects due to resonance scattering, including interference, result in asymmetric line shapes and deviations of the NO product quantum state distribution from the statistical prediction. As an example, when $\mathrm{NO}_{2}$ is photodissociated $235 \mathrm{~cm}^{-1}$ above the dissociation limit, Figure 7 shows that the experimentally measured $\operatorname{NO}\left({ }^{2} \Pi_{1 / 2}\right)$ rotational distributions fluctuate about the average statistical prediction when the $\mathrm{A}^{\prime}$ and $\mathrm{A}^{\prime \prime} \Lambda$-doublet states of $\mathrm{NO}$ are detected state specifically, but agree well with phase space theory when the populations in the two $\Lambda$-doublet states are summed.

While the experiments above give examples of mode-selective product internal state distributions, excitation in a structured electronic absorption spectrum can also result in mode-selective branching between two dissociation product channels. A particularly interesting case is the ${ }^{2} \mathrm{~A}_{2}$ photodissociation of $\mathrm{OClO},{ }^{154}$ where the branching beween the $\mathrm{Cl}+\mathrm{O}_{2}$ and $\mathrm{ClO}+$ $\mathrm{O}$ channels varies dramatically and systematically with the nature of the vibrational band excited in the absorption. The selective dissociation dynamics evolves on more than one Born-Oppenheimer potential energy surface in this system, so the complicated mechanism for the selectivity is still being investigated.

The review of investigations of statistical theories using photoexcitation to excited electronic states would not be complete without mentioning the beautiful studies of intramolecular vibrational energy redistribution in electronically excited molecules. Chemical timing experiments, ${ }^{155}$ which disperse the fluroescence from vibronic state selectively excited molecules immersed in a variable pressure of $\mathrm{O}_{2}$ quencher gas, allow the 
measurement of intramolecular vibrational energy redistribution times from a Franck-Condon bright mode into coupled vibrational states of the bath. Recent experiments ${ }^{156}$ have shown that when one replaces one of the $\mathrm{F}$ atoms in $p$-diflurobenzene with a methyl group, the dephasing of the bright mode occurs 40 times faster. This increase and the two- to four-fold increase upon deuteration of the methyl group can be accounted for by the increase in the density of coupled states that the internal rotation-vibration interaction turns on. However, when the methyl group is moved from the para to the meta position, the IVR rate increases by a factor of 10 , an acceleration that cannot be accounted for by the twofold increase in the density of coupled states that results from the decrease in vibrational symmetry. One must conclude that there is an increase in the coupling strength between the internal rotation and molecular vibration when the methyl group is moved to the meta position. These studies allow very detailed analysis of vibrational energy redistribution in state-selected vibronically excited molecules.

3. Using Photodissociation To Probe the Breakdown of the Born-Oppenheimer Separation of Nuclear and Electronic Motion. Much of our predictive ability for the rates and dynamics of chemical reaction pathways has relied on statistical transition state theories ${ }^{157-159}$ or, in smaller systems, quantum scattering calculations ${ }^{160}$ on a single adiabatic potential energy surface. The potential energy surface gives the energetic barriers to each chemical reaction and allows prediction of the reaction rates. However, the chemical reaction dynamics evolves on a single potential energy surface only if the Born-Oppenheimer separation of nuclear and electronic motion is valid. ${ }^{161,162}$ The paragraphs below give examples of recent photodissociation experiments on classes of chemical reactions in which the failure of the adiabatic approximation is critical in determining the dynamics of the chemical reaction and the branching between energetically allowed chemical reaction channels. Several of the experiments described below focus on systems where the reactive trajectories cross the transition state of the chemical reaction in the vicinity of a conical intersection, a process long understood to render the adiabatic approximation invalid. Others demonstrate the importance of considering the possibility of nonadiabaticity at the transition state of any chemical reaction with a barrier along the reaction coordinate, including ground state bimolecular reactions.

A simple but chemically important consequence of the breakdown of the Born-Oppenheimer approximation is the marked change in the expected branching between energetically allowed chemical bond fission channels due to nonadiabatic recrossing of a reaction barrier. Statistical transition state theories predict that, given comparable preexponential factors, the reaction pathway with the lowest energetic barrier will dominate. However, because barriers result from avoided electronic configuration crossings, the electronic wave function changes rapidly along the reaction coordinate near the saddle point. This is exactly the situation in which the adiabatic approximation may fail. Semiclassically, the nuclear dynamics can unexpectedly turn back from the barrier at energies well above the barrier and thus markedly reduce the reaction rate.

Recent photofragment velocity and angular distribution experiments on the competition between $\mathrm{C}-\mathrm{Cl}$ and $\mathrm{C}-\mathrm{Br}$ fission in bromoacetyl and bromopropionyl chloride at $248 \mathrm{~nm}$ 163 demonstrate how such nonadiabatic recrossing can alter the branching ratio between bond fission channels. Although statistical transition state theories predict that $\mathrm{C}-\mathrm{Br}$ fission, the reaction pathway with the lowest energetic barrier on the ${ }^{1} \mathrm{~A}^{\prime \prime}$ surface, should dominate, the experiments find the $\mathrm{C}-\mathrm{Cl}$ bond cleaves preferentially, arriving at a $\mathrm{C}-\mathrm{Br}: \mathrm{C}-\mathrm{Cl}$ fission branch-
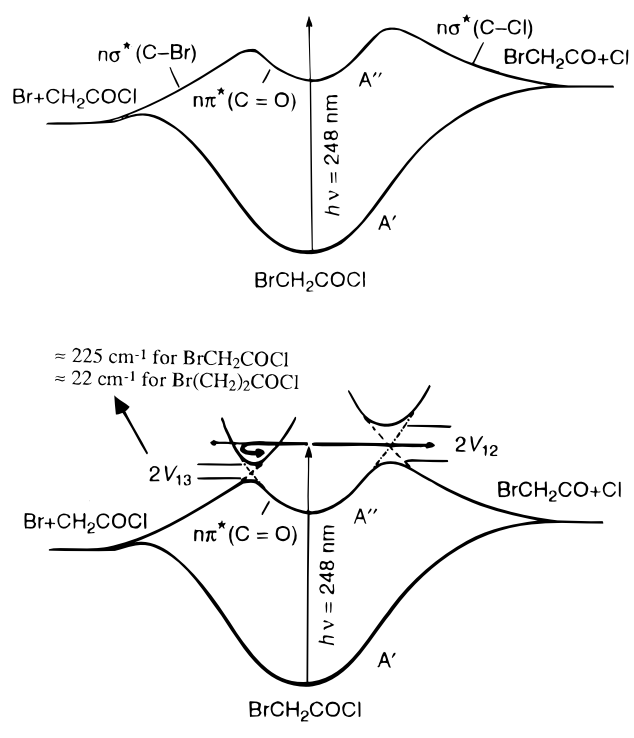

$\mathrm{C}-\mathrm{Br} \longleftarrow$ reaction coordinate $\longrightarrow \mathrm{C}-\mathrm{Cl}$

Figure 8. Schematic reaction coordinates for $\mathrm{C}-\mathrm{Cl}$ and $\mathrm{C}-\mathrm{Br}$ fission from the $248 \mathrm{~nm}$ photodissociation of $\mathrm{Br}\left(\mathrm{CH}_{2}\right)_{n} \mathrm{COCl}$. The lower frame shows the splitting, determined from configuration interaction single excitation calculations at $r_{\mathrm{C}=\mathrm{O}}=1.188 \AA$ (for $\mathrm{BrCH}_{2} \mathrm{COCl}$ ) and 1.195 $\AA$ (for $\mathrm{Br}\left(\mathrm{CH}_{2}\right)_{2} \mathrm{COCl}$ ) between the two $\mathrm{A}^{\prime \prime}$ potential energy surfaces at the avoided crossing along the $\mathrm{C}-\mathrm{Br}$ bond fission coordinate. Adapted with permission from Figures 1 and 11 of ref $163 \mathrm{~d}$.

ing ratio of 0.04:1 in bromoacetyl chloride and at most 0.05:1 in bromopropionyl chloride from molecules excited to the ${ }^{1}\left(\mathrm{n}, \pi^{*}(\mathrm{C}=\mathrm{O})\right) 1^{1} \mathrm{~A}^{\prime \prime}$ potential energy surface.

Crude $a b$ initio calculations support a model in which $\mathrm{C}-\mathrm{Br}$ fission is suppressed by the inability of the electronic wave function to keep up with nuclear motion across the barrier to $\mathrm{C}-\mathrm{Br}$ fission, a barrier resulting from the avoided crossing of the $\mathrm{n}_{\mathrm{O}} \pi^{*}(\mathrm{C}=\mathrm{O})$ and the $\mathrm{n}_{\mathrm{pBr}} \sigma^{*}(\mathrm{C}-\mathrm{Br})$ configurations. The calculations also show why nonadiabatic recrossing so markedly suppresses $\mathrm{C}-\mathrm{Br}$ fission in bromopropionyl chloride. Figure 8 shows that the splitting at the avoided crossing along the $\mathrm{C}-\mathrm{Br}$ fission reaction coordinate is reduced from typically $200 \mathrm{~cm}^{-1}$ in bromoacetyl chloride to only $20 \mathrm{~cm}^{-1}$ in brompropionyl chloride; ${ }^{163 \mathrm{~d}}$ a simple Landau-Zener calculation estimates that over $99.9 \%$ of the trajectories that try to cross the low-energy barrier to $\mathrm{C}-\mathrm{Br}$ fission in brompropionyl chloride undergo a nonadiabatic transition to the upper adiabat instead of crossing the barrier and resulting in $\mathrm{C}-\mathrm{Br}$ fission. Thus, any prediction based on relative barrier heights on the adiabatic potential energy surface is grossly in error. This and subsequent experiments showed that a particular class of reactions is particularly susceptible to nonadiabatic effects due to the small splitting bewteen adiabats at the reaction barrier. This class of reaction is one in which individual orbital symmetry is not conserved along the adiabatic reaction coordinate. ${ }^{164}$

Recent photodissociation experiments ${ }^{129}$ on $\mathrm{CH}_{3} \mathrm{SH}$ afford the opportunity to investigate nonadiabaticity in the transition state region of bimolecular reactions. In the saddle point region of a typical $\mathrm{AB}+\mathrm{C} \rightarrow \mathrm{A}+\mathrm{BC}$ reaction shown in Figure 9, the electronic wave function changes most rapidly with nuclear coordinate, so the Born-Oppenheimer approximation may fail, resulting in a nonadiabatic transition to the upper surface in the region of the saddle point. Photodissociation of $\mathrm{CH}_{3} \mathrm{SH}$ offers the chance to directly access both the upper and lower adiabats, respectively, near the transition state region of the excited state $\mathrm{CH}_{3} \mathrm{~S}+\mathrm{H} \rightarrow \mathrm{CH}_{3}+\mathrm{SH}$ bimolecular reaction to probe the influence of nonadiabatic coupling in chemical reaction dynamics. When the molecule is excited at $193 \mathrm{~nm}$ in 


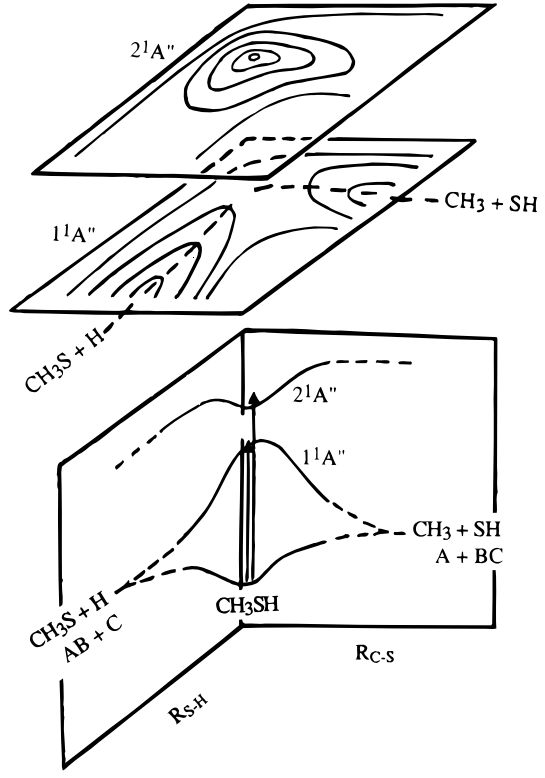

Figure 9. Schematic contour plots of the excited electronic potential energy surfaces for $\mathrm{CH}_{3} \mathrm{SH}$, showing the two lowest ${ }^{1} \mathrm{~A}$ " adiabatic excited state surfaces. The lower diagram shows the reaction coordinate for the bimolecular reaction $\mathrm{A}+\mathrm{BC} \rightarrow \mathrm{AB}+\mathrm{C}$ along the lower excited state surface. The Franck-Condon region, marked by $\mathrm{X}$, lies near the saddle point on the lower excited state surface. Reproduced with permission from ref 129. Copyright 1993 American Institute of Physics.

the higher energy absorption band to the upper bound adiabat, it dissociates via nonadiabatic coupling to the transition state region of the lower adiabat. Photofragment velocity and angular distribution measurements on $\mathrm{CH}_{3}-\mathrm{S}-\mathrm{H}$ show the nonadiabatic decay to the transition state region of the lower surface occurs in less than a picosecond and results in a factor of 8 larger branching to decay of the transition state complex to the $\mathrm{CH}_{3}$ + SH exit channel than did direct excitation to the lower adiabat at $222 \mathrm{~nm} .{ }^{129,130,165}$ The larger branching ratio results from the stretching of the $\mathrm{C}-\mathrm{S}$ bond on the upper adiabat, evidenced in emission spectrocopy experiments. ${ }^{125}$ This dynamics allows the molecule to sample a region of the transition state upon nonadiabatic transition to the lower dissociative surface that enhances $\mathrm{C}-\mathrm{S}$ bond fission over that obtained by accessing the transition state directly via Franck-Condon excitation from the ground state. As good ab initio potential energy surfaces are being developed for this system, ${ }^{166}$ dynamics calculations are seeking to understand how to accurately describe the dynamics, ${ }^{167}$ and workers are identifying regions of the potential surfaces in which nonadiabatic transitions occur efficiently. ${ }^{168}$

Although the experiments above remind us that in general it is near the transition state region that the Born-Oppeneheimer approximation may fail, for many reactions the approximation is a very good one. The approximation's validity depends on the regions of the reactive potential energy surface sampled by the reactive trajectories. This is evident in systems like the $\mathrm{H}$ $+\mathrm{O}_{2}$ reaction, which at thermal energies in the ground electronic state occurs adiabatically, but at energies accessed by reacting a $2.3 \mathrm{eV} \mathrm{H}$ atom with $\mathrm{O}_{2}{ }^{169}$ is subject to failure of the BornOppenheimer separation of nuclear and electronic motion because the trajectories can then sample regions of the potential energy surface near the conical intersections in linear and T-shaped geometries. ${ }^{170}$

Several recent photodissociation experiments have elucidated the importance of considering what regions of the potential energy surface the reactive trajectories access in determining whether nonadiabatic effects will be important. For instance, in the $\tilde{\mathrm{A}}$ state photodissociation of both $\mathrm{NH}_{3}$ and $\mathrm{CH}_{3} \mathrm{NH}_{2}$ there

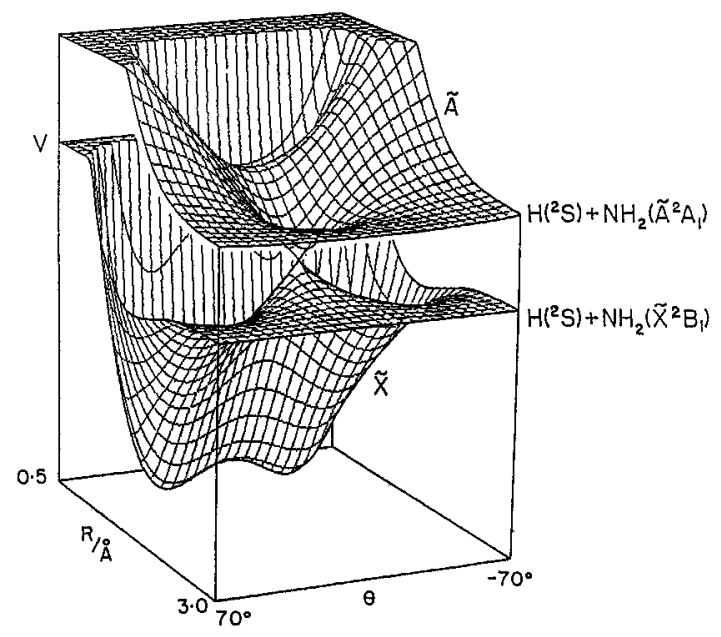

Figure 10. Ground and first excited state potential energy surfaces for ammonia, showing the region of intersection along the $\mathrm{N}-\mathrm{H}$ bond fission coordinate (or $\mathrm{C}-\mathrm{N}$ bond fission coordinate in $\mathrm{CH}_{3} \mathrm{NH}_{2}$ ) as it varies with the out-of-plane angle $\theta$. Adapted with permission from ref 171.

is a conical intersection along the $\mathrm{N}-\mathrm{H}$ and $\mathrm{C}-\mathrm{N}$ fission reaction coordinates, respectively. If the bond fission proceeds adiabatically as the trajectories traverse the conical intersection, excited state $\mathrm{NH}_{2} \tilde{\mathrm{A}}\left({ }^{2} \mathrm{~A}_{1}\right)$ product is formed. Diabatic traversal of the conical intersection results in a transition from the upper to the lower adiabat shown in Figure $10,{ }^{171}$ producing ground state $\mathrm{NH}_{2}$ product. Since nonadiabatic transitions occur efficiently at conical intersections, one expects a significant fraction of ground state $\mathrm{NH}_{2}$ products. However, while $\mathrm{N}-\mathrm{H}$ fission in ammonia results in a mixture of the two products at energies where both exit channels are energetically allowed, $\mathrm{C}-\mathrm{N}$ fission in $\mathrm{CH}_{3} \mathrm{NH}_{2}$ produces only excited state $\mathrm{NH}_{2} \cdot{ }^{172}$ To understand how the dissociative trajectories manage to traverse the reaction coordinate adiabatically despite the conical intersection, one must consider the detailed dynamics that the molecule undergoes. Upon Franck-Condon excitation to the upper adiabat, where the equilibrium geometry is planar rather than pyramidal about the $\mathrm{N}$ atom, $\mathrm{NH}_{2}$ wagging motion ensues, as evidenced by the structure in the absorption spectrum assigned to a progression in the $\mathrm{NH}_{2}$ wag in $\mathrm{CH}_{3} \mathrm{NH}_{2} \cdot{ }^{173}$ As the molecule traverses the conical intersection with considerable energy in $\mathrm{NH}_{2}$ wagging motion, the amplitude of the dissociative wave function is small at planar geometries and large at bent geometries at the outer turning points of the wagging motion, so there is little amplitude near the point of conical intersection in Figure 10. Classically, then, trajectories traverse the conical intersection mainly at bent geometries where the splitting between the upper and lower adiabats is large, and the system thus remains on the upper adiabat throughout the dissociation and results in excited state $\mathrm{NH}_{2}$ product.

Other experiments have sought to control whether the dissociative trajectories traverse the conical intersection at geometries near the point of conical intersection, or at bent geometries where the splitting is larger, so as to control the resulting branching between adiabatic and diabatic dissociation products. In $\mathrm{CX}_{3} \mathrm{I}(\mathrm{X}=\mathrm{H}, \mathrm{D}, \mathrm{F})^{174}$ and $\mathrm{ICN},{ }^{175-177}$ if one photoexcites molecules with one or more quanta in the bend, the dissociative trajectories traverse the conical intersection at bent geometries where the splitting between adiabats is larger, resulting in an increase in the branching to the adiabatic reaction products. A somewhat different example of the sensitivity of nonadiabatic effects to the region the surface accessed by the molecular wave function occurs in the photodissociation of HCO. In that system, excited state resonances whose wave 
functions sample linear geometries on the upper of two RennerTeller coupled surfaces, thus nearing the seam of electronic degeneracy with the lower dissociative surface, evidence much larger widths due to their fast nonadiabatic decay onto the lower Born-Oppenheimer potential energy surface. ${ }^{178-181}$ Overall, the study of nonadiabatic effects in chemical reaction dynamics is one of the most current and active areas of investigation, challenging our dearest assumptions.

\section{Summary}

This article has attempted to provide an overview of the field of photodissociation dynamics by summarizing the tremendous experimental advances that have been made during the past 30 years and by describing several of the conceptual issues that are of current interest in the field. The experimental effort in photodissociation has been one of the most productive in all of physical chemistry. In spite of the fact that photodissociation is a "mature" area of research, qualitative advances still occur with considerable regularity, to the extent that experiments are now routinely being performed which would have been inconceivable (or at least very difficult!) five years ago. On the conceptual side, experimental results combined with theoretical advances in the area of dynamics and quantum chemistry are providing a detailed perspective on the multitude of pathways by which an electronically excited molecule can dissociate. This is enabling us to move beyond the simple models often used to describe photodissociation dynamics and, instead, to gain a deep understanding of the interplay between electronic and nuclear motion that underlies all of photodissociation. Given that this level of understanding has only been achieved for a handful of molecules, we expect the field of photodissociation dynamics to be a fruitful one for many years to come.

Acknowledgment. This article is dedicated to our children, Ellyn (L.J.B.) and Griffith (D.M.N.), both of whom were born during the course of writing the manuscript. We hope that this article will not be hopelessly outdated by the time they are old enough to read it. L. J. Butler's salary during the preparation of this review article was supported by the National Science Foundation under Grant CHE-9307500. D. M. Neumark acknowledges support from the Director, Office of Basic Energy Sciences, Chemical Sciences Division of the U.S. Department of Energy, under Contract DE-AC03-76SF00098. D.M.N. also thanks JILA at the University of Colorado, Boulder, for its hospitality while this paper was being written.

\section{References and Notes}

(1) Busch, G. E.; Mahoney, R. T.; Morse, R. I.; Wilson, K. R. J. Chem. Phys. 1969, 51, 449.

(2) Riley, S. J.; Wilson, K. R. Discuss. Faraday Soc. 1972, 53, 132.

(3) Busch, G. E.; Cornelius, J. F.; Mahoney, R. T.; Morse, R. I.; Schlosser, D. W.; Wilson, K. R. Rev. Sci. Instrum. 1970, 41, 1066. Kent Wilson tells the authors of this review that "The precursor to our work, and a very important one, was Dick Zare's thesis, ${ }^{7}$ in which he discussed beautifully the theory of photodissociation and angular distributions and certainly inspired my thinking about how to build an apparatus to measure such things." Yuan Lee's crossed beam apparatuses followed this apparatus in time, introducing differential pumping and an improved mass spectrometer detector that is incorporated in most modern photofragmentation apparatuses, along with a rotatable source or detector to facilitate detecting slowly recoiling photofragments.

(4) See the first photofragment angular distribution (measured by the photofragments etching a film coated on a hemispherical surface), work by a graduate student of R. Bersohn in: Solomon, J. J. Chem. Phys. 1967, 47, 889 .

(5) Karplus, S. Molecular Photodissociation Processes. Ph.D. Thesis, Columbia University, 1965.

(6) Schnieder, L.; Meier, W.; Welge, K. H.; Ashfold, M. N. R.; Western, C. M. J. Chem. Phys. 1990, 92, 7027.
(7) Zare, R. N. Mol. Photochem. 1972, 4, 1. See also early work in: Zare, R. N. Molecular Fluorescence and Polarization. Ph.D. Thesis, Harvard University, 1964.

(8) For a review of using a "universal detector" in molecular beam apparatuses, see: Lee, Y. T.; McDonald, J. D.; LeBreton, P. R.; Herschbach, D. R. Rev. Sci. Instrum. 1969, 40, 1402. Lee et al. introduced a differentially pumped, straight-through mass spectrometer detector that revolutionized the field.

(9) (a) Busch, G. E.; Wilson, K. R. J. Chem. Phys. 1972, 56, 3638. (b) Yang, S. C.; Bersohn, R. J. Chem. Phys. 1974, 61, 4400.

(10) For a recent example, see: Kash, P. W.; Waschewsky, G. C. G.; Morss, R. E.; Butler, L. J.; Francl, M. M. J. Chem. Phys. 1994, 100, 3463.

(11) Sabety-Dzvonik, M. J.; Cody, R. J. J. Chem. Phys. 1977, 66, 125.

(12) Cody, R. J.; Sabety-Dzvonik, M. J.; Jackson, W. M. J. Chem. Phys. 1977, 66, 2145. Halpern, J. B.; Jackson, W. M. J. Phys. Chem. 1982, 86, 973.

(13) Lengel, R. K.; Zare, R. N. J. Am. Chem. Soc. 1978, 100, 7495

(14) Danon, J.; Filseth, S. V.; Feldmann, D.; Zacharias, H.; Dugan, C. H.; Welge, K. H. Chem. Phys. 1978, 29, 345.

(15) McDonald, J. R.; Miller, R. G.; Baronavski, A. P. Chem. Phys. Lett. 1977, 51, 57.

(16) Drozdoski, W. S.; Baronavski, A. P.; McDonald, J. R. Chem. Phys. Lett. 1979, 64, 421.

(17) Yang, S. C.; Freedman, A.; Kawasaki, M.; Bersohn, R. J. Chem. Phys. 1980, 72, 4058

(18) Hawkins, W. G.; Houston, P. L. J. Chem. Phys. 1980, 73, 297.

(19) Roellig, M. P.; Houston, P. L.; Asscher, M.; Haas, Y. J. Chem. Phys. 1980, 73, 5081

(20) Zacharias, H.; Geilhaupt, M.; Meier, K.; Welge, K. H. J. Chem. Phys. 1981, 74, 218 .

(21) Pfab, J.; Hager, J.; Krieger, W. J. Chem. Phys. 1983, 78, 266

(22) Andresen, P.; Ondrey, G. S.; Titze, B. Phys. Rev. Lett. 1983, 450, 486. Andresen, P.; Ondrey, G. S.; Titze, B.; Rothe, E. W. J. Chem. Phys. 1984, 80, 2548.

(23) Ondrey, G.; van Veen, N.; Bersohn, R. J. Chem. Phys. 1983, 78, 3732.

(24) Vasudev, R.; Zare, R. N.; Dixon, R. N. J. Chem. Phys. 1984, 80, 4863

(25) Hawkens, W. G.; Houston, P. L. J. Chem. Phys. 1982, 76, 729.

(26) Qian, C. X. W.; Noble, M.; Nadler, I.; Reisler, H.; Wittig, C. J. Chem. Phys. 1985, 83, 5573.

(27) Bamford, D. J.; Filseth, S. V.; Foltz, M. F.; Hepburn, J. W.; Moore, C. B. J. Chem. Phys. 1985, 82, 3032.

(28) Nesbitt, D. J.; Petek, H.; Foltz, M. F.; Filseth, S. V.; Bamford, D. J.; Moore, C. B. J. Chem. Phys. 1985, 83, 223

(29) Miller, R. L.; Kable, S. H.; Houston, P. L.; Burak, I. J. Chem. Phys. 1992, 96, 332.

(30) Waller, I. M.; Davis, H. F.; Hepburn, J. W. J. Phys. Chem. 1987, 91, 506. Waller, I. M.; Hepburn, J. W. J. Chem. Phys. 1988, 88, 6658.

(31) Buntin, S. A.; Cavanaugh, R. R.; Richter, L. J.; King, D. S. J. Chem. Phys. 1991, 94, 7937

(32) Butenhoff; T. J.; Carleton, K. L.; Moore, C. B. J. Chem. Phys. 1990, 92, 377

(33) Cassassa, M. P.; Foy, B. R.; Stephenson, J. C.; King, D. S. J. Chem. Phys. 1991, 94, 250.

(34) Chen, J.; Dagdigian, P. J. J. Chem. Phys. 1994, 100, 4884.

(35) Terentis, A. C.; Knepp, P. T.; Kable, S. H. J. Phys. Chem. 1995 99, 12704

(36) Chamberlain, G. A.; Simons, J. P. Chem. Phys. Lett. 1975, 32, 355; J. Chem. Soc., Faraday Trans. 2 1975, 71, 2043.

(37) Greene, C. H.; Zare, R. N. Annu. Rev. Phys. Chem. 1982, 33, 119;

J. Chem. Phys. 1983, 78, 674 .

(38) Radhakrishnan, G.; Ng, D.; Estler, R. C. Chem. Phys. Lett. 1981, $84,260$.

(39) Mons, M.; Dimicoli, I. Chem. Phys. 1989, 130, 307.

(40) Hradil, V. P.; Suzuki, T.; Hewitt, S. A.; Houston, P. L.; Whitaker,

B. J. J. Chem. Phys. 1993, 99, 4455.

(41) Powis, I.; Black, J. F. J. Phys. Chem. 1989, 93, 2461.

(42) Chandler, D. W.; Thoman, Jr., J. W.; Janssen, M. H. M.; Parker, D. H. Chem. Phys. Lett. 1989, 156, 151. Chandler, D. W.; Janssen, M. H. M.; Stolte, S.; Strickland, R. N.; Thoman, Jr., J. W.; Parker, D. H. J. Phys. Chem. 1990, 94, 4839.

(43) Ogorzalek Loo, R.; Strauss, C. E.; Haerri, H.-P.; Hall, G. E.; Burak, I.; Hepburn, J. W. J. Chem. Soc., Faraday Trans. 2 1989, 85, 1185.

(44) Kim, D. Y.; Brandstater, N.; Pipes, L.; Garner, T.; Baugh, D. J. Phys. Chem. 1995, 99, 4364.

(45) Rinnen, K.-D.; Buntine, M. A.; Kliner, D. A. V.; Zare, R. N.; Huo, W. M. J. Chem. Phys. 1991, 95, 214.

(46) Steadman, J.; Baer, T. J. Chem. Phys. 1989, 91, 6113.

(47) He, G.; Yang, Y.; Huang, Y.; Hashimoto, S.; Gordon, R. J. J. Chem. Phys. 1995, 103, 5488.

(48) Cromwell, E. F.; Liu, D.-J.; Vrakking, M. J. J.; Kung, A. H.; Lee, Y. T. J. Chem. Phys. 1991, 95, 297.

(49) Venkataraman, B. K.; Valentini, J. J. Chem. Phys. Lett. 1992, 194, 191. 
(50) Chu, J. J.; Marcus, P.; Dagdigian, P. J. Chem. Phys. 1990, 93, 257. (51) Huang, Y.; Yang, Y.; He, G.; Gordon, R. J. J. Chem. Phys. 1993, $99,2752$.

(52) Hall, G. E.; Sears, T. J.; Frye, J. M. J. Chem. Phys. 1989, 90, 6234. (53) Suzuki, T.; Kanamori, H.; Hirota, E. J. Chem. Phys. 1991, 94, 6607. (54) Moore, D. S.; Bomse, D. S.; Valentini, J. J. J. Chem. Phys. 1983, 79, 1745. Levene, H. B.; Nieh, J.-C.; Valentini, J. J. J. Chem. Phys. 1987, $87,2583$.

(55) Valentini, J. J.; Gerrity, D. P.; Phillips, D. L.; Nieh, J.-C.; Tabor, K. D. J. Chem. Phys. 1987, 86, 6745.

(56) Debarre, D.; Lefebvre, M.; Pealat, M.; Taran, J.-P. E. J. Chem. Phys. 1985, 83, 4476.

(57) Zahedi, M.; Harrison, J. A.; Nibler, J. W. J. Chem. Phys. 1994, $100,4043$.

(58) Donaldson, D. J.; Leone, S. R. Chem. Phys. Lett. 1986, 132, 240.

(59) Woodbridge, E. L.; Fletcher, T. R.; Leone, S. R. J. Phys. Chem. 1988, 92,5387 .

(60) Fletcher, T. R.; Leone, S. R. J. Chem. Phys. 1988, 88, 4720

(61) Fletcher, T. R.; Leone, S. R. J. Chem. Phys. 1989, 90, 871.

(62) Woodbridge, E. L.; Ashfold, M. N. R.; Leone, S. R. J. Chem. Phys.

1991, 94, 4195. Lindner, J.; Lundberg, J. K.; Williams, R. M.; Leone, S.

R. Rev. Sci. Instrum. 1995, 66, 2812.

(63) Simons, J. P. J. Phys. Chem. 1987, 91, 5378

(64) Houston, P. L. J. Phys. Chem. 1987, 91, 5388

(65) Schmeidl, R.; Dugan, H.; Meier, W.; Welge, K. H. Z. Phys. A 1982, 304,137

(66) Xu, Z; Koplitz, B; Buelow, S.; Baugh, D; Wittig, C. Chem. Phys. Lett. 1986, 127, 534. Xu, Z.; Koplitz, B.; Wittig, C. J. Chem. Phys. 1987, 87,1062 .

(67) Satyapal, S.; Johnson, G. W.; Bersohn, R.; Oref, I. J. Chem. Phys. 1990, 93, 6398.

(68) Matsumi, Y.; Tonokura, K.; Kawasaki, M.; Inoue, G.; Satyapal,

S.; Bersohn, R. J. Chem. Phys. 1991, 94, 2669.

(69) Mo, Y.; Tonokura, K.; Matsumi, Y.; Kawasaki, M.; Sato, T.;

Arikawa, T.; Reilly, P. T. A.; Xie, Y.; Yang, Y.; Huang, Y.; Gordon, R. J.

J. Chem. Phys. 1992, 97, 4815.

(70) Dubs, M.; Huber, J. R. Chem. Phys. Lett. 1984, 108, 123.

(71) Nadler, I.; Mahgerefteh, M.; Reisler, H.; Wittig, C. J. Chem. Phys. 1985, 82,3885

(72) Dubs, M.; Bruhlman, U.; Huber, J. R. J. Chem. Phys. 1986, 84, 3106.

(73) Hall, G. E.; Sivakumar, N.; Houston, P. L.; Burak, I. Phys. Rev. Lett. 1986, 56, 1671 .

(74) Docker, M.; Hodgson, A.; Simons, J. P. Chem. Phys. Lett. 1986, 128,264

(75) Gericke, K.-H.; Klee, S.; Comes, F. J.; Dixon, R. N. J. Chem. Phys.

1986, 85,4463 .

(76) Dixon, R. N. J. Chem. Phys. 1986, 85, 1866

(77) Hall, G. E.; Houston, P. L. Annu. Rev. Phys. Chem. 1989, 40, 375.

(78) Sivakumar, N.; Hall, G. E.; Houston, P. L.; Hepburn, J. W.; Burak,

I. J. Chem. Phys. 1988, 88, 3692 .

(79) Docker, M. P.; Ticktin, A.; Bruhlamann, U.; Huber, J. R. J. Chem.

Soc., Faraday Trans. 2 1989, 85, 1169

(80) Burak, I.; Hepburn, J. W.; Sivakumar, N.; Hall, G. E.; Chawlac,

G.; Houston, P. L. J. Chem. Phys. 1987, 86, 1258.

(81) Docker, M. P.; Hodgson, A.; Simons, J. P. Faraday Discuss. Chem. Soc. 1986, 82, 163.

(82) Gericke, K.-H.; Theinl, R.; Comes, F. J. J. Chem. Phys. 1990, 92, 6548

(83) Carleton, K. L.; Butenhoff, T. J.; Moore, C. B. J. Chem. Phys.

1990, 93, 3907.

(84) Novicki, S. W.; Vasudev, R. J. Chem. Phys. 1990, 93, 8725

(85) Brouard, M.; Duxon, S.; Enriquez, P. A.; Simons, J. P. J. Chem.

Soc., Faraday Trans. 1993, 89, 1435.

(86) Hall, G. E.; Wu, M. J. Phys. Chem. 1993, 97, 10911. Wu, M.;

Hall, G. E. J. Photochem. Photobiol. A: Chem. 1994, 80, 45.

(87) Kades, E.; Rosslein, M.; Bruhlmann, U.; Huber, J. R. J. Phys. Chem. 1993, 97, 989.

(88) Uberna, R.; Cline, J. I. J. Chem. Phys. 1995, 102, 4705

(89) Mons, M.; Dimicoli, I. Chem. Phys. Lett. 1986, 131, 298.

(90) Ogarzalek Loo, R.; Hall, G. E.; Haerri, H.-P.; Houston, P. L. J.

Phys. Chem. 1988, 92, 5.

(91) Mons, M.; Dimicoli, I. J. Chem. Phys. 1989, 90, 4037

(92) Trentelman, K. A.; Kable, S. H.; Moss, D. B.; Houston, P. L. J.

Chem. Phys. 1989, 91, 7498.

(93) Chandler, D. W.; Houston, P. L. J. Chem. Phys. 1987, 87, 1445. (94) Heck, A. J. R.; Chandler, D. W. Annu. Rev. Phys. Chem. 1995, $46,335$.

(95) Suits, A. G.; Miller, R. L.; Bontuyan, L. S.; Houston, P. L. J. Chem.

Soc., Faraday Trans. 1993, 89, 1443.

(96) Deshmukh, S.; Myers, J. D.; Xantheas, S. S.; Hess, W. P. J. Phys. Chem. 1994, 98, 12535.

(97) Kinugawa, T.; Arikawa, T. J. Chem. Phys. 1992, 96, 4801.

(98) Hess, W. P.; Chandler, D. W.; Thoman, Jr., J. W. Chem. Phys. 1992, 163, 277.
(99) Suzuki, T.; Tonokura, K.; Bontuyan, L. S.; Hashimoto, N. J. Phys. Chem. 1994, 98, 13447

(100) Hertz, R. A.; Syage, J. A. J. Chem. Phys. 1994, 100, 9265

(101) Miller, R. L.; Suits, A. G.; Houston, P. L.; Toumi, R.; Mack, J.

A.; Wodtke, A. M. Science (Washington, D.C.) 1994, 265, 1831.

(102) Janssen, M. H. M.; Parker, D. H.; Sitz, G. O.; Stolte, S.; Chandler, D. W. J. Phys. Chem. 1991, 95, 8007.

(103) Suits, A. G.; Miller, R. L.; Bontuyan, L. S.; Houston, P. L. J. Chem. Soc., Faraday Trans. 1993, 89, 1443.

(104) Mastenbroek, J. W. G.; Taatjes, C. A.; Nauta, K.; Janssen, M. H

M.; Stolte, S. J. Phys. Chem. 1995, 99, 4360. 13637

105) Sanov, A.; Bieler, C. R.; Reisler, H. J. Phys. Chem. 1995, 99,

(106) Drabbels, M.; Morgan, C. G.; McGuire, D. S.; Wodtke, A. M. J. Chem. Phys. 1995, 102, 611.

(107) Zhang, Q.; Kandel, S. A.; Wasserman, T. A. W.; Vaccaro, P. H J. Chem. Phys. 1992, 96, 1640. Vaccaro, P. In Molecular Dynamics and Spectroscopy by Stimulated Emission Pumping; Dai, H. L., Field, R. W.,

Eds.; World Scientific: Singapore, 1995; pp 1-71.

(108) Buntine, M. A.; Chandler, D. W.; Hayden, C. C. J. Chem. Phys. 1992, 97, 707 .

(109) Butenhoff, T. J.; Rohlfing, E. A. J. Chem. Phys. 1993, 98, 5460; 1993, $98,5469$.

(110) Wasserman, T. A. W.; Arias, A. A.; Kandel, S. A.; Hsu, D.; Vaccaro, P. H. Proc. SPIE 1995, 2548, 220.

(111) Continetti, R. E.; Cyr, D. R.; Osborn, D. L.; Leahy, D. J.; Neumark,

D. M. J. Chem. Phys. 1993, 99, 2616.

(112) Leahy, D. J.; Osborn, D. L.; Cyr, D. R.; Neumark, D. M. J. Chem. Phys. 1995, 103, 2495.

(113) Osborn, D. L.; Leahy, D. J.; Ross, E. M.; Neumark, D. M. Chem. Phys. Lett. 1995, 235, 484.

(114) Cyr, D. R.; Continetti, R. E.; Metz, R. B.; Osborn, D. L.; Neumark,

D. M. J. Chem. Phys. 1992, 97, 4937.

(115) Cyr, D. R.; Leahy, D. J.; Osborn, D. L.; Continetti, R. E.; Neumark,

D. M. J. Chem. Phys. 1993, 99, 8751 .

(116) Osborn, D. L.; Choi, H.; Neumark, D. M. Adv. Chem. Phys., in press.

(117) Shapiro, M.; Bersohn, R. Annu. Rev. Phys. Chem. 1982, 33, 409.

(118) (a) Heller, E. J. J. Chem. Phys. 1978, 68, 2066. (b) Heller, E. J.

J. Chem. Phys. 1978, 68, 3891. (c) Heller, E. J. In Potential Energy Surfaces and Dynamics Calculations; Truhlar, D. G., Ed.; Plenum Press: New York,

1981. (d) Tannor, D. J.; Heller, E. J. J. Chem. Phys. 1982, 77, 202.

(119) Dixon, R. N. Chem Soc. Rev. 1994, 23, 375.

(120) See review of time-dependent methods in: Schinke, R. Photodissociation Dynamics; University Press: Cambridge, England, 1993; Chapter 4. Also, a review of time-independent methods in Photodissociation Dynamics, Chapter 3.

(121) Herzberg, G. Molecular Spectra and Molecular Structure: III. Electronic Spectra and Electronic Structure of Polyatomic Molecules; Van Nostrand: Princeton, 1966.

(122) Henriksen, N. E.; Zhang, J.; Imre, D. G. J. Chem. Phys. 1988, 89,5607

(123) Imre, D.; Kinsey, J. L.; Sinha, A.; Krenos, J. J. Phys. Chem. 1984, 88,3956

(124) See early work by Lao et al. (Lao, K. Q.; Person, M. D.; Xayariboun, P.; Butler, L. J. J. Chem. Phys. 1990, 92, 823) and associated theory of Harris et al. (Harris, R. A.; Wedlock, M. R.; Butler, L. J.; Freed, K. F. J. Chem. Phys. 1992, 96, 2437)

(125) Stevens, R. E.; Kittrell, C.; Kinsey, J. L. J. Phys. Chem. 1995, 99, 11067.

(126) Browning, P. W.; Jensen, E.; Waschewsky, G. C. G.; Tate, M. R.; Butler, L. J.; Hessler, J. P. J. Chem. Phys. 1994, 101, 5652 and theoretical references within.

(127) Heumann, B.; Schinke, R. J. Chem. Phys. 1994, 101, 7488.

(128) Bradforth, S. E.; Weaver, A.; Arnold, D. W.; Metz, R. B.; Neumark, D. M. J. Chem. Phys. 1990, 92, 7205.

(129) Jensen, E.; Keller, J. S.; Waschewsky, G. C. G.; Stevens, J. E.; Graham, R. L.; Freed, K. F.; Butler, L. J. J. Chem. Phys. 1993, 98, 2882.

(130) Keller, J. S.; Kash, P. W.; Jensen, E.; Butler, L. J. J. Chem. Phys. 1992, 96, 4324.

(131) Wilson, S. H. S.; Ashfold, M. N. R.; Dixon, R. N. J. Chem. Phys.

1994, 101, 7538

(132) Segall, J.; Wen, Y.; Singer, R.; Dulligan, M.; Wittig, C. J. Chem. Phys. 1993, 99, 6600.

(133) Butler, L. J. Chem. Phys. Lett. 1991, 182, 393

(134) Person, M. D.; Lao, K. Q.; Eckholm, B. J.; Butler, L. J. J. Chem.

Phys. 1989, 91, 812

(135) Brudzynski, R. J.; Sension, R. J.; Hudson, B. Chem. Phys. Lett. 1990, 165, 487.

(136) (a) Van Veen, G. N. A.; Mohamed, K. A.; Baller, T.; De Vries, A. E. Chem. Phys. 1983, 74, 261. (b) Continetti, R. E.; Balko, B. A.; Lee, Y. T. Chem. Phys. Lett. 1991, 182, 400. (c) Xie, X.; Schneider, L.; Wallmeier, H.; Boettner, R.; Welge, K. H. J. Chem. Phys. 1990, 92, 1608. (d) Weiner, B. R.; Levene, H. B.; Valentini, J. L.; Baronavski, A. P. J. Chem. Phys. 1989, 90, 1403. 
(137) (a) Goursaud, S.; Sizun, M.; Fiquet-Fayard, F. J. Chem. Phys. 1976, 65, 5453. (b) Fiquet-Fayard, F.; Sizun, M.; Abgrall, H. Chem. Phys. Lett. 1976, 37, 72

(138) Engel, V.; Schinke, R. J. Chem. Phys. 1988, 88, 6831.

(139) Shafer, N.; Satyapal, S.; Bersohn, R. J. Chem. Phys. 1989, 90, 6807

(140) (a) Vander Wal, R. L.; Scott, J. L.; Crim, F. F. J. Chem. Phys.

1990, 92, 803. (b) Bar, I.; Cohen, Y.; David, D.; Rosenwaks, S.; Valentini,

J. J. J. Chem. Phys. 1990, 93, 2146. (c) Vander Wal, R. L.; Scott, J. L.;

Crim, F. F.; Weide, K.; Schinke, R. J. Chem. Phys. 1991, 94, 3548. (d)

Bronikowski, M. J.; Simpson, W. R.; Girard, B.; Zare, R. N. J. Chem. Phys. 1991, 95, 8647.

(141) Soep, B.; Whitham, C. J.; Keller, A.; Visticot, J. P. Faraday Discuss. Chem. Soc. 1991, 91, 191.

(142) Waller, I. M.; Kitsopoulos, T. N.; Neumark, D. M. J. Phys. Chem. 1990, 94, 2240

(143) Ogai, A.; Brandon, J.; Reisler, H.; Suter, H. U.; Huber, J. R.; von Dirke, M.; Schinke, R. J. Chem. Phys. 1992, 96, 6643.

(144) For a similar system, see mapping of HONO transition-state wave functions in: Schinke, R.; Untch, A.; Suter, H. U.; Huber, J. R. J. Chem. Phys. 1991, 94, 7929.

(145) Schulz, P. A.; Sudbo, Aa. S.; Krajnovich, D. J.; Kwok, H. S.; Shen, Y. R.; Lee, Y. T. Annu. Rev. Phys. Chem. 1979, 30, 379.

(146) Freed, K. F.; Nitzan, A. In Energy Storage and Redistribution in Molecules; Hinze, J., Ed.; Plenum: New York, 1976; p 467.

(147) Crim, F. F. Annu. Rev. Phys. Chem. 1984, 35, 657

(148) Uzer, T. Phys. Rep. (Review Section of Phys. Lett.) 1991, 199, 73.

(149) Green, W. H., Jr.; Moore, C. B.; Polik, W. F. Annu. Rev. Phys. Chem. 1992, 43, 591 and extensive references within.

(150) (a) Butler, L. J.; Hintsa, E. J.; Shane, S. F.; Lee, Y. T. J. Chem. Phys. 1987, 86, 2051. (b) Butler, L. J.; Hintsa, E. J.; Lee, Y. T. J. Chem. Phys. 1986, 84, 4104

(151) Stevens, J. E.; Kitchen, D. C.; Waschewsky, G. C. G.; Butler, L. J. J. Chem. Phys. 1995, 102, 3179.

(152) Schinke, R.; Keller, H.-M.; Stumph, M.; Dobbyn, A. J. J. Phys. B: At. Mol. Opt. Phys. 1995, 28, 3081.

(153) (a) Robie, D. C.; Hunter, M.; Bates, J. L.; Reisler, H. Chem. Phys. Lett. 1992, 192, 279. (b) Hunter, M.; Reid, S. A.; Robie, D. C.; Reisler, H. J. Chem. Phys. 1993, 99, 1093. (c) Reid, S. A.; Brandon, J. T.; Hunter, M.; Reisler, H. J. Chem. Phys. 1993, 99, 4860. (d) Reid, S. A.; Robie, D. C.; Reisler, H. J. Chem. Phys. 1994, 100, 4256. (e) Reid, S. A.; Reisler, H. J. Chem. Phys. 1994, 101, 5683.

(154) Davis, H. F.; Lee, Y. T. J. Phys. Chem. 1992, 96, 5681.

(155) Coveleskie, R. A.; Dolson, D. A.; Parmenter, C. S. J. Phys. Chem. 1985, 89, 645 .

(156) (a) Moss, D. B.; Parmenter, C. S. J. Chem. Phys. 1993, 98, 6897.

(b) Timbers, P. J.; Parmenter, C. S.; Moss, D. B. J. Chem. Phys. 1994, 100,1028 .
(157) Robinson, P. J.; Holbrook, K. A. Unimolecular Reactions; Wiley-Interscience: London, 1972.

(158) Laidler, K. J.; King, M. C. J. Phys. Chem. 1983, 87, 2657.

(159) Truhlar, D. L.; Hase, W. L.; Hynes, J. T. J. Phys. Chem. 1983 , 87, 2664.

(160) See, for example, extensive references on pages 296-299 in: Levine, R. D.; Bernstein, R. B. Molecular Reaction Dynamics and Chemical Reactivity; Oxford University Press: New York, 1987; Section 5.6. (161) Born, M.; Oppenheimer, R. Ann. Phys. 1927, 84, 457.

(162) Tully, J. C. In Dynamics of Molecular Collisions; Miller,W. H., Ed.; Plenum: New York, 1976; Part B, p 217.

(163) (a) Person, M. D.; Kash, P. W.; Schofield, S. A ; Butler, L. J. J. Chem. Phys. 1991, 95, 3843. (b) Person, M. D.; Kash, P. W.; Butler, L. J. J. Chem. Phys. 1992, 97, 355. (c) Kash, P. W.; Waschewsky, G. C. G.; Butler, L. J. J. Chem. Phys. 1994, 100, 4017. (d) Kash, P. W.; Waschewsky, G. C. G.; Butler, L. J.; Francl, M. M. J. Chem. Phys. 1993, 99, 4479. (164) Waschewsky, G. C. G.; Kash, P. W.; Myers, T. L.; Kitchen, D.

C.; Butler, L. J. J. Chem. Soc., Faraday Trans. 1994, 90, 1581.

(165) Vaghjiani, G. L. J. Chem. Phys. 1993, 99, 5936.

(166) Stevens, J. E.; Freed, K. F.; Arendt, M. F.; Graham, R. L. J. Chem. Phys. 1994, 101, 4832.

(167) Stevens, J. E.; Jang, H. W.; Butler, L. J.; Light, J. C. J. Chem. Phys. 1995, 102, 7059.

(168) Yarkony, D. R. J. Chem. Phys. 1994, 100, 3639.

(169) Kim, H. L.; Wickramaaratchi, M. A.; Zheng, X.; Hall, G. E. J. Chem. Phys. 1994, 101, 2033

(170) Kendrick, B.; Pack, R. T. J. Chem. Phys. 1995, 102, 1994

(171) Biesner, J.; Schnieder, L.; Ahlers, G.; Xie, X.; Welge, K. H.; Ashfold, M. N. R.; Dixon, R. N. J. Chem. Phys. 1989, 91, 2901.

(172) Waschewsky, G. C. G.; Kitchen, D. C.; Browning, P. W.; Butler, L. J. J. Phys. Chem. 1995, 99, 2635.

(173) Tsuboi, M.; Hirakawa, A. Y.; Kawashima, H. J. Mol. Spectrosc. 1969, 29, 216

(174) Person, M. D.; Kash, P. W.; Butler, L. J. J. Chem. Phys. 1991, 94, 2557

(175) Kash, P. W.; Butler, L. J. J. Chem. Phys. 1992, 96, 8923.

(176) Qian, J.; Tannor, D. J.; Amatatsu, Y.; Morokuma, K. J. Chem. Phys. 1994, 101, 9597.

(177) Bowman, J. M.; Mayrhofer, R. C.; Amatatsu, Y. J. Chem. Phys. 1994, 101, 9469

(178) Tanaka, K.; Davidson, E. R. J. Chem. Phys. 1979, 70, 2904

(179) Dixon, R. N. Mol. Phys. 1985, 54, 333.

(180) Loison, J. -C.; Kable, S. H.; Houston, P. L. J. Chem. Phys. 1991, 94, 1796

(181) Goldfield, E. M.; Gray, S. K.; Harding, L. B. J. Chem. Phys. 1993, 99, 5812 .

JP953518B 